\title{
28 Research Square \\ Gene Correlation Network Analysis To Identify Regulatory Factors In Sciatic Nerve Injury
}

\section{Liuxun Li ( $\square$ LucienLi01@163.com )}

Third Affiliated Hospital of Sun Yat-Sen University

\section{Xiaokang Du}

Third Affiliated Hospital of Sun Yat-Sen University

\section{Haiqian Ling}

Third Affiliated Hospital of Sun Yat-Sen University

\section{Yuhang Li}

Third Affiliated Hospital of Sun Yat-Sen University

\section{Xuemin Wu}

Shenzhen Hospital of Southern Medical University

\section{Anmin Jin}

Zhujiang Hospital

\section{Meiling Yang}

Shenzhen Hospital of Southern Medical University

\section{Research Article}

Keywords: sciatic nerve injury, weighted gene co-expression network analysis, gene set enrichment analysis, protein-protein interaction, bioinformatics

Posted Date: June 24th, 2021

DOI: https://doi.org/10.21203/rs.3.rs-625536/v1

License: () (i) This work is licensed under a Creative Commons Attribution 4.0 International License. Read Full License 


\section{Abstract}

Background: Sciatic nerve injury (SNI), which frequently occurs under the traumatic hip and hip fracture dislocation, induces serious complications such as motor and sensory loss, muscle atrophy, or even disabling. The present work aimed to determine the regulating factors and gene network related to the SNI pathology.

Methods: Sciatic nerve injury dataset GSE18803 with 24 samples was randomly divided into adult group and neonate group. We performed weighted gene co-expression network analysis (WGCNA) to identify modules associated with SNI in the two groups. Moreover, differentially expressed genes (DEGs) were determined from every group, separately. Subsequently, co-expression network, protein-protein interaction (PPI) network, enrichment analysis and gene set enrichment analysis (GSEA) were integrated to identify hub genes and associated pathways. GSE30165 was used as the test set for investigating the hub gene involvement within SNI. Finally, we employed DGIdb for predicting the possible therapeutic agents leading to the abnormal up-regulation of hub genes.

Results: 14 SNI status modules and 97 DEGs were identified in adult group, while 15 modules and 21 DEGs in neonate group. A total of 12 hub genes was overlapping from co-expression and PPI network. After the results from both test and training sets were overlapped, we verified that the ten real hub genes showed remarkably up-regulation within SNI. According to functional enrichment of DEGs, the above genes participated in the immune effector process, inflammatory responses, the antigen processing and presentation, and the phagocytosis. GSEA also supported that gene sets with the highest significance was mostly related to the cytokine-cytokine receptor interaction.

Conclusions: The gene expression network is determined in the present work based on the related regulating factors within SNI, which sheds more lights on SNI pathology and offers the possible biomarkers and therapeutic targets in subsequent research.

\section{Introduction}

Sciatic nerve injurie (SNI) are one of common peripheral nerve injury (PNI), which often cause severe disability, decreased life quality, as well as tremendous social and economic burdens (1). SNI usually result from a variety of trauma (2,3), hip arthroplasty (4), autoimmune diseases (5), and musculoskeletal diseases. Nerve injuries of traumatic and iatrogenic etiologies can lead to dramatic neurological functional loss. However, even though the best medical treatment is applied, the neurological function recovery is difficult to predict. Nonetheless, there are still numerous barriers to be solved to attain nerve injury recovery, including target innervation specificity, low regeneration rate, target end-organ degeneration and segmental nerve defect following the extended denervation period. Most existing studies have investigated the morphologies of injury and regeneration of peripheral nerves (6-8). Notably, it may be of greater importance to analyze the changes in gene expression during the injury and regeneration of peripheral nerves, so as to examine the molecular foundation for such morphological 
changes as well as modulation of local microenvironment. However, the changes in gene expression together with the associated biological processes in the processes of injury and regeneration of peripheral nerves remain largely unknown. Consequently, to better promote the prevention and treatment of $\mathrm{PNI}$, it is necessary to shed more lights on molecular mechanisms that regulate peripheral nerve regeneration at a broader level.

Fortunately, impaired peripheral nerves have an opportunity to regenerate by themselves (9). It has been previously suggested that, peripheral axonal transection leads to sufficient changes of the respective regeneration ability, metabolism, excitability, survival, different intrinsic and extrinsic signal sensitivities, and transmitter function $(10,11)$. Additionally, PNI exhibits stereotypic histopathological reactions, which indicate that the harmonious gene expression procedure is activated (12). As a result, numerous articles have examined the numbers of genes with up-regulation or down-regulation expression following PNI (13, 14). Previous research has used transcriptome analysis in rats to report that dynamic alterations in core genes and biological processes may take place within sciatic nerve stumps in the process of nerve regeneration $(15,16)$. However, approaches adopted in such articles usually examine individual genes, whereas genes exert their functions through the co-expression gene network showing consistent biological functions in vivo. It is speculated that identification of such co-expression patterns can shed more lights on the disease-related biological pathways. WGCNA is a new tool for analyzing the gene expression patterns of various samples (17). WGCNA may be used for gene set analysis based on highly synergistic changes and possible biomarkers, which are adopted to identify therapeutic targets corresponding to gene sets with phenotypes. Genes clustered within adjacent groups of a network can be regarded as modules, while those with the closest connection are referred to as "hubs". WGCNA has been extensively utilized in genomic research, such as glioblastoma (18), Kawasaki disease (19), schizophrenia spectrum (20), and so on.

In the present study, weighted gene co-expression network analysis (WGCNA) was employed to analyze the hub genes and pathways involved in SNI pathogenic mechanism in rats of different ages. Then, the expression levels of core genes were detected using a training set and a test set, respectively. Subsequently, Reactome, Gene Ontology (GO) functional annotations, together with Kyoto Encyclopedia of Genes and Genomes (KEGG) pathway enrichment were adopted for investigating molecular mechanisms underlying SNI. This study identified the novel significant biomarkers to explore the mechanism of SNI from a bioinformatics perspective, thereby facilitating SNI diagnosis and treatment. The flow chart of the study design is presented in Fig. 1.

\section{Methods}

\section{Search strategy and eligibility criteria}

In the present study, we obtained the mRNA expression data of sciatic nerve injury (SNI) based on GEO database by use of keywords "sciatic nerve injury" in NCBI database (http://www.ncbi.nlm.nih.gov/geo/) until June 10, 2021. The search strategy of the study was designed as follows: (a) datasets of gene 
expression profiles concerning the use of the microarray chip technique; (b) studies that compared the expression data in SNI samples versus non-SNI samples. Studies not satisfying the above-mentioned criteria were eliminated. Two reviewers searched the database independently.

\section{Establishment of co-expression network and analysis of module functions}

Firstly, DEGs expression profiles were examined for screening the appropriate genes and samples. Secondly, the R language 'WGCNA' package was employed to establish the co-expression network of DEGs $(21,22)$. Afterwards, each pair-wise gene was functioned using Pearson's correlation matrix. Thirdly, we adopted the power function $\mathrm{amn}=|\mathrm{cmn}| \beta$ (amn represents the adjacency of gene $\mathrm{m}$ relative to gene $\mathrm{n}$, whereas $\mathrm{cmn}$ stands for Pearson correlation of gene $\mathrm{m}$ with gene $\mathrm{n}$ ) to create the weighted adjacency matrix,while $\beta$ was the soft threshold factor adopted to stress the strong associations across genes and to penalize those weak relationships. Fourth, topological overlap matrix (TOM) adjacency was converted for measuring the gene network connectivity deemed to be the total value of the adjacency to the remaining genes in generating the network. The mean linkage hierarchical clustering was created by the dissimilarity measure based on TOM, and the minimal size (gene group) was set at 50 for gene dendrogram. Thereafter, genes that had akin expression patterns were clustered as the same gene module. Lastly, the module eigengene dissimilarity was determined. Then, such gene modules were performed functional enrichment analyses to identify the SNI related modules. The above gene modules were conducted functional enrichment analysis for identifying related modules affecting SNI in rats of different ages.

\section{Identification of SNI status hub module}

For identifying modules showing significant associations with illness state traits (SNI vs. NON-SNI), MEs (which represent first principle component in one module) (17) were associated with the external traits to identify correlations with the highest significance. In addition, Gene significance (GS) determines the absolute value associations between genes and external characteristics. Meanwhile, module membership (MM) suggests the association of gene expression profiles with MEs. In this study, genes that showed the greatest GS and MM values in the interested modules were identified as the natural candidates in later analysis (23-26).

\section{Hub genes validation}

A hub gene is substantially related to other genes within the module, which is suggested in previous studies to display functional significance. Firstly, this study screened hub genes within the co-expression network from the SNI phenotype-related module. Besides, we imported DEGs into the Search Tool for the Retrieval of Interacting Genes/Proteins (STRING) (https://string-db.org/) (27), and the confidence $>0.4$ was chosen for creating the protein-protein interaction (PPI) network. Later, Cytoscape (www.cytoscape.org/) (28) was carried out for PPI network visualization. Subsequently, the Cytoscape plug-in CytoHubba was adopted for calculating every protein node degree. Nodes that had great connectivity degree were more important to maintain the network stability. Any gene within PPI network 
with the connectivity of $\geq 6$ (node/edge) was screened to be the hub gene. Later, we deemed those shared PPI network and co-expressed hub genes as the "real" hub genes, which were selected for subsequent analysis. The Venn diagram was constructed using Venny 2.1.0 (https://bioinfogp.cnb.csic.es/tools/venny/index.html) for visualizing those common hub genes in PPI and co-expression networks between adult group and neonate group.

\section{Gene ontology and KEGG pathway enrichment analyses after SNI}

GO analysis has been developed as an efficient way to carry out functional enrichment in a large scale. Besides, KEGG is also an extensively applied database that preserves excessive data on drugs, chemical substances, diseases, biological processes, and signaling pathways. In the current work, the Metascape software (http://metascape.org) (29) was employed for GO and KEGG analyses on the DEGs. $P<0.05$ indicated statistical significance. Besides, Reactome knowledgebase (https://reactome.org/) (30-32) offers the detailed molecular data for signaling, DNA replication, transport, metabolism, along with additional cell processes as the well-organized molecular transformation network, and it represents the modified version for the classical metabolic map within the single consistent data model. In the present study, Reactome knowledgebase (https://reactome.org/) (30-32) was employed to identify 10 most significant biological functions.

\section{Gene set enrichment analysis}

GSEA (https://software.broad institute.org/gsea/index.jsp) (33) has been developed as a computation approach on the basis of genesets (namely, gene groups with shared biological functions). GSEA was adopt to investigate the enrichment of previously determined biological processes within the DEGsderived gene rank. In line with gene expression levels, samples from both adult group and neonate group were separately classified as SNI samples and NON-SNI samples. Terms enriched in each gene were recognized with the thresholds for nominal $P<0.05$ and false discovery rate (FDR) $q<0.25$.

\section{Identification of the potential drugs}

Drug Gene Interaction Database (DGIdb) (version 4.2.0, https://www.dgidb.org) (34), one of the openly accessible database, covers information on drug-gene interaction, drug sensitive and targeted genome. In this study, DGIdb was searched for predicting the possible hub gene-interacting molecule compounds or drugs and visualizing the drug-gene interaction network through using Cytoscape.

\section{Results}

\section{Included study characteristics}

GSE30165 and GSE18803 microarray datasets were obtained from GEO database $(16,35)$. Of them, GSE30165 dataset contained was obtained on the basis of platform GPL7294 Agilent-014879 Whole Rat Genome Microarray 4x44K G4131F, whereas the GSE18803 contained microarray data acquired from ipsilateral dorsal horns at 7 days following sham-operation or SNI surgery in both groups (12 from 10- 
day-old (neonate) group and 12 from 8-12-week-old (adult) group) on the basis of platform GPL341 [RAE230A] Affymetrix Rat Expression 230A Array. There were 6 sham-operation and 6 SNI samples from every age group collected on the basis of GPL341 [RAE230A] Affymetrix Rat Expression 230A Array. In the present study, GSE18803 dataset was utilized to construct co-expression and PPI networks in both neonate group and adult group for identifying "real" hub genes and pathways. The GSE30165 microarray data was obtained from proximal sciatic nerve samples $(0.5 \mathrm{~cm})$ and L4-6 dorsal root ganglia samples at $0,1,4,7$ and 14 days following sciatic nerve removal. The GSE30165 dataset was used as the test set for result validation in the current study.

\section{DEGs identification}

We utilized R language "limma" package to identify DEGs within the GSE18803 microarrays. DEGs were identified with the two criterions: $\mid \log 2$ fold change $(F C) \mid \geq 1$ and the $P$ value $\leq 0.05$. In adult group, we identified altogether 97 DEGs within SNI samples relative to normal samples, among which, 96 showed up-regulation while 1 showed down-regulation. And In neonate group, 21 DEGs were discovered to be differentially expressed between SNI and NON-SNI samples, and all these DEGs were up-regulated. Benjamini-Hochberg correction was utilized for adjusted P-values. A volcano plot for DEGs from this microarray is shown in Fig. 2A-B.

\section{Weighted co-expression network construction and hub module analysis}

The "WGCNA" package in R language was adopted for constructing the gene co-expression networks. Then, we obtained a total 9,371 genes. The 5,000 most significant genes that showed the greatest standard deviations (SDs) were chosen to perform hierarchical cluster, group similar expression levels into modules, and select Power $\beta=16$ to ensure a scale-free network. It was observed that, 12 samples were basically classified as 2 clusters. In addition, the Pearson's correlation was also carried out. In total, 14 modules were excavated, and the pink module was the most tightly related with SNI traits (Fig. 3A-B). Thereafter, the interactions among these 14 modules were also examined, followed by the plotting of a network heatmap. According to these findings, every module served as an independent validation for one another, demonstrating the high level of independence across various modules, as well as the relative gene expression independence for every module. For purposes of exploring co-expression similarity among these 14 modules, eigengene connectivity was assessed, and then consensus correlation was subjected to clustering analysis (Fig. 3C). In addition, intramodular analysis including MM (module significance) and GS (gene significance) was performed in those 14 modules. The pink module was excavated to further explore the highly related genes. Figure 3D displays the scatter plots for GS regarding SNI traits, together with illness state compared with MM within the pink module. In the case of SNI, GS and MM were significantly positively correlated, which suggested that the pink module elements with the greatest importance (central) might show high correlation with such external traits. Furthermore, to evaluate the influence of different ages in SNI status, the co-expression networks were similar measured using WGCNA software package in neonate group, separately. Finally, 15 modules were mined, 
and the lightcyan module was found to be most tightly associated with SNI traits in neonate group (Fig. 4A-D).

\section{Identification of SNI core genes between hub modules and PPI networks in different ages groups}

Subsequently, the DEGs-associated PPI network was used to identify 63 hub genes at the thresholds of connectivity $\geq 6$ and confidence $>0.4$ in adult group. In addition, 13 PPI networks related hub genes were screened out using the similar thresholds in neonate group. The more strict factors were used in additional analyses, including module connectivity determined through absolute Pearson's correlation coefficient (cor.geneModuleMembership >0.8), together with relationships of clinical characteristics determined based on absolute Pearson's correlation coefficient (cor.geneTraitSignifcance $>0.2$ ). In adult group, there were 481 highly connected genes identified in the pink module. In contrast, 88 co-expression related hub genes were discovered to be highly connected in lightcyan module in neonate group. Based on these analyses, 12 hub genes (C1qb, C1qa, C1qc, Tyrobp, Fcer1g, Cd74, Fcgr2a, Mpeg1, C4a, Aif1, RT1$\mathrm{A} 2$, and $\mathrm{C} 3$ ) related with SNI were detected in both the coexpression and PPI networks (Fig. 5A).

According to our results, each hub gene was upregulated. Therefore, the above 12 genes were identified to be real hub genes to indicate SNI status, which were screened in later analyses (Fig. 5B).

\section{Validation of hub genes}

To investigate hub genes related with SNI, the expression levels of C1qb, C1qa, C1qc, Tyrobp, Fcer1g, Cd74, Fcgr2a, Mpeg1, C4a, Aif1, RT1-A2, and C3 were detected using the training set GSE18803 dataset and the test set GSE30165 dataset, respectively. In the training set, we found all hub genes had statistically significant differences in SNI group (Fig. 6A-7I). In the test set, all hub genes except C1qb and RT1-A2 were significantly upregulated in the SNI groups in comparison with the non-SNI groups (Fig. 7A$8 \mathrm{I})$. After overlapping the results from the training set and test set, we found ten hub genes (C1qa, C1qc, Tyrobp, Fcer1g, Cd74, Fcgr2a, Mpeg1, C4a, Aif1, and C3) were altered in the comparison between the SNI and normal control samples.

\section{Gene ontology and KEGG pathway enrichment analyses after SNI}

For better understanding gene functions in hub genes, we adopted the Metascape software for GO functional annotation and KEGG enrichment. Based on our results, "inflammatory response" was the gene set with the highest significance (Fig. 9A). The analysis also showed that SNI was associated with immune effector process, antigen processing and presentation, and phagocytosis. Meanwhile, as revealed by KEGG results, DEGs were mainly associated with antigen processing and presentation, osteoclast differentiation, and staphylococcus aureus infection pathways (Fig. 9B). For validating the biofunctions related to such hub genes, the other method was adopted. Later, Reactome, the approach for functional enrichment analysis, was applied in aligning targets with the corresponding biological functions. Thereafter, pathways were presented in the bubble chart based on the Entities found, Entities 
ratio, together with Entities FDR functions. As a result, the SNI samples showed remarkable relationships with immune system, innate immune system, neutrophil degranulation, and cytokine signaling in immune system (Fig. 9C). Besides, those 10 most significant functional pathways were sorted according to the entities. As observed from the bar chart, those resultant pathways exerted vital parts in immune system (Fig. 9D).

\section{GSEA analysis}

This study conducted GSEA for identifying the potential mechanism underlying SNI. Samples were divided into SNI vs. NON-SNI in both adult group and neonate group, respectively. The analysis indicated that the most significant-enriched gene sets positively correlated with SNI in adult group, which included the cytokine-cytokine receptor interaction, the chemokine signaling pathway, and the $T$ cell receptor signal transduction pathway (Fig. 10A). In addition, significant gene sets with the highest enrichment level showed positive correlation with SNI in neonate group, were the cytokine-cytokine receptor interaction, the chemokine signaling pathway, and the Fc gamma R-mediated phagocytosis (Fig. 10B). The common pathways in both adult group and neonate group are the cytokine-cytokine receptor interaction, and the chemokine signaling pathway.

\section{Identification of the potential drugs}

This study used DGIdb for determining the possible molecular compounds or drugs with the effects on reversing the increased expression of hub genes within SNI. According to the drug-gene interaction network (Fig. 11), we identified 7 molecular compounds or drugs, including metyldopa, copper and zinc chloride, and they showed differential regulation of C3 and C1QC expression. Moreover, milatuzumab was detected to interact with CD74. In addition, 10 molecular compounds or drugs, like adalimumab, adalimumab or etanercept, were identified to show interaction with FCGR2A. Furthermore, 5 molecular compounds or drugs (including etanercept) modulated C1QA, whereas 2 including aspirin and benzylpenicilloyl polylysine modulated FCER1G.

\section{Discussion}

$\mathrm{SNI}$ has the same feature as other frequently occurring but refractory diseases (such as immune and inflammatory responses that prolong disease course), which is related to several complications. At present, the changes and remodelling taking place in myelin sheaths and axons are increasingly understood; as a result, more and more studies have been conducted to explore the immune response and inflammation related to PNI, and it is suggested that several elements and factors are involved in this process. Growing evidence suggests that the success in neurological functional recovery is determined by debris removal and axonal regeneration rates (Wallerian degeneration) following nerve injury $(36,37)$. Upon nerve injury, the innate immune responses of PNS (like cytokine release magnitude and timing) play important role in Wallerian degeneration (5). Immediate impacts on macrophages following injury will have certain effects on the entire biochemical event cascade taking place following nerve injury; in this regard, the direct stimulation of axonal growth by use of nerve growth factors may be unnecessary. 
However, the molecular mechanisms on how nerve regeneration and precisely how significant neuromuscular sequelae and complications are improvement have not been completely understood yet, at least partially.

In the present study in which an integrated bioinformatical study on SNI was performed, an overlap method was employed to combine WGCNA, PPI network, and GSEA for identifying the hub genes as well as associated pathways. As suggested by our results, the pink module in adult group and lightcyan module in neonate group were recognized to be of clinical significance by WGCNA. In later analyses, 12 genes between co-expression and PPI networks in both adult group and neonate group were identified to be the real hub genes, which indicated the potentially vital roles of such genes during SNI occurrence and development. Subsequently, to investigate hub genes in SNI of different ages, the expression levels of the 12 genes were detected using a training set and a test set, respectively. Collectively, 10 real hub genes (C1qa, C1qc, Tyrobp, Fcer1g, Cd74, Fcgr2a, Mpeg1, C4a, Aif1, and C3) in both adult group and neonate group revealed significant differences between training set and test set.

We also conducted further potential function and pathway enrichment for clarifying the DEGs functions. According to $G O$ analysis, DEGs related to SNI were mainly associated with inflammatory response, immune effector process, antigen processing and presentation, and phagocytosis. Consistent with KEGG enrichment analyses, the antigen processing and presentation was a significant pathway. In addition, supported in Reactome analyses, the SNI samples showed dramatically relationships with immune system, innate immune system, neutrophil degranulation, and cytokine signaling in immune system. In addition, GSEA supported that gene sets with statistical significance were mostly related to immune responses. Conforming to this work, previous studies confirmed that SNI was highly associated with inflammation $(38,39)$. Immunocytes, including lymphocytes, resident cells, neutrophils, and macrophages, can produce various chemical molecules, including purines, lipids, histamine, protons, bradykinin, serotonin, chemokines, cytokines, nerve growth factors in the process of inflammation (40). It is interesting to note that certain mediating factors show direct sensitization on nociceptors, which results in neuropathic pain (41). These results are in line with previous studies. In the case of SNI, immune response is postponed at first, then the continuous hyperinflammatory state is detected, accompanying with the reduced repair process (42). The inflammatory response was mostly associated with immune response usually related to lymphocytes, neutrophils and macrophages. Leukocytic infiltration may exert a certain part in catabolic enzyme generation and inflammatory response, causing the disrupted structure and function of nerve tissues. The peripheral nervous system may regrow their axons after an injury, but such capability is affected by the extracellular environment and inherent regrowing ability for supporting regrowth. Chemokines can influence neuronal differentiation, proliferation and nerve regeneration, and their expression increased in the case of inflammation and injury (43). Nonetheless, numerous immunocytes and inflammatory factors are related to the regulation of continuous tissue damage responses, which enhances tissue repair (44). Collectively, sciatic nerve injury and nerve regeneration display intricate biological processes, involving in the coordination of inflammatory response and immunoregulatory signals after peripheral nerve injury. 
For better verifying the associations between hub genes and SNI, we obtained hub gene expression profiles based on the GEO database. The 10 genes enrolled from the above-mentioned database, including C1qa, C1qc, Tyrobp, Fcer1g, Cd74, Fcgr2a, Mpeg1, C4a, Aif1, and C3 were found to be higher in $\mathrm{SNI}$ as compared to the non-SNI between adult group and neonate group. These indicated that these 10 core genes were significantly associated with $\mathrm{SNI}$ at both adult and neonate ages. More and more studies on transcriptomic analysis in vitro and in vivo verify that $\mathrm{Cd} 74$ played a vital part in the progression of sciatic nerve injury (45-48). Linnartz-Gerlach B et al. (49) reported that Tyrobp mutations or genetic variants were associated with the aging-related inflammatory neurodegenerative disorders. Another research conducted WGCNA on the expression profiles of genes specific to aging and cell-type in mice, which identified hub genes including $\mathrm{C} 1 \mathrm{qa}$, Tyrobp, and Fcer1g as the critical players related to neurodegenerative disorders and aging in humans (50). According to Wang $\mathrm{J}$ and colleagues (51), C1qc and Fcer1g facilitated neuropathic pain occurrence following SNI through the defense and immune pathways. C4a is related to immune responses at each level and additional events like organ regeneration and neural development (52). Huelsenbeck SC et al. (53) reported that C3 peptide enhanced the functional motor recovery and axonal regeneration following PNI. As for, C1qa, C1qc, Tyrobp, Fcer1g, Fcgr2a, Mpeg1, C4a, and Aif1, they are relatively new molecules with only few reports regarding their role in SNI at present. Nevertheless, they played an important role in SNI and were significantly different between normal samples and SNI. The above genes shed more lights on clinical and experimental studies. Nonetheless, more investigation is needed to completely understand their functions in SNI.

For predicting the candidate efficient treatment against SNI and the associated concurrent diseases, this study used DGIdb database for determining the therapeutic agents showing effects on reversing the abnormal up-regulation of SNI-associated hub genes. Tumor necrosis factor-alpha (TNF-a) is suggested to exert a vital part during demyelination and apoptosis, while blocking its expression enhances neural healing (54). According to previous reports, TNF- $a$ antagonists are effective on Schwann cells and axons within SNI, and TNF-a is related to the modulation of axonal regeneration (55). An increasing number of epidemiological studies have suggested that, the anti-TNF-a therapies, including adalimumab, etanercept and adalimumab, are utilized to treat different peripheral nerve diseases, including chronic inflammatory demyelinating polyneuropathy, Miller Fisher syndrome, Guillain-Barré syndrome, mononeuropathy multiplex, multifocal motor neuropathy accompanied by conduction block, and axonal sensorimotor polyneuropathy (56). Adalimumab was detected as the efficient neuroprotective drug to heal the nerves in PNI model of rats, particularly in the early phase (54). Trastuzumab have also been reported to enhance peripheral nerve regeneration following repair from acute and chronic PNI $(57,58)$. More studies are needed to explore the functions of the above-mentioned molecular compounds and drugs within SNI, together with the corresponding concurrent diseases as the candidate therapeutic targets.

Nonetheless, certain limitations should still be noted. First, this work identified numerous new pathways related to SNI, but it was still restricted due to the intrinsic biases of enrichment analysis and the microarray data available. Second, this study obtained the open-sourced data, but data quality was not assessed; besides, it adopted the uncommonly utilized Affymetrix gene expression arrays. Third, Mus musculus-derived tissue samples of training set were different from the Rattus norvegicus-derived 
samples of test set, and this might lead to diverse target genes in the 2 organisms following nerve injury. Therefore, if the database has samples updates, more studies are warranted in the future. Fourth, laboratory experiments should be carried out to verify our results. Cells isolated from SNI samples needed to be cultured in vitro for determining the related molecular mechanisms of hub gene expression. Thus, the gene knockdown preclinical animal models can help to examine the identified gene functions and evaluate their functions in SNI. Fifth, to increase the result reliability, we need more samples for repeated measurements. Last, enrichment analysis was also limited in identifying pathways because the gene lists verified might lead to over-representation of the well-identified pathways. As a result, the functions of such hub genes as well as pathways within SNI, and the functional meaning in SNI development should be validated in more research.

\section{Conclusion}

To sum up, through a series of integrated bioinformatics analyses, we screened a total of 10 hub genes with verified high expression within $\mathrm{SNI}$, and predicted potential therapeutic agents associated with the progression of SNI. According to our findings, some pathways related to SNI conformed to the known knowledge regarding disease pathology. Results in this study can shed more lights on biological pathways related to $\mathrm{SNI}$ and identify some possible regulating factors as the interventional targets. Nonetheless, more research is required to verify the association of hub gene functions with the immune responses during SNI development.

\section{Abbreviations}

SNI: Sciatic nerve injury; WGCNA: Weighted gene co-expression network analysis; DEG: Differentially expressed gene; PPI: Protein-protein interaction; GESA: Gene Set Enrichment Analysis; DGIdb: Drug Gene Interaction Database; PNI: Peripheral nerve injury; GO: Gene Ontology; KEGG: Kyoto Encyclopedia of Genes and Genomes; GEO: Gene Expression Omnibus; TOM: Topological overlap matrix; GS: Gene significance; MM: Module membership; STRING: Search Tool for the Retrieval of Interacting Genes; FDR: False discovery rate; C1qb: Complement C1q B chain; C1qa: Complement C1q A chain; C1qc:

Complement C1q subcomponent subunit C; Tyrobp: Transmembrane immune signaling adaptor TYROBP; Fcer1g: Fc fragment Of IgE receptor Ig; Cd74: CD74 molecule; Fcgr2a: Fc fragment of IgG receptor Ila; Mpeg1: Macrophage expressed gene 1; C4a: Complement C4A; Aif1: Allograft inflammatory factor 1; RT1A2: RT1 class I, A2; C3: Complement C3; TNF-a: Tumor necrosis factor-alpha.

\section{Declarations}

\section{Acknowledgements}

We acknowledge GEO database for providing their platforms and contributors for uploading their meaningful datasets. 


\section{Authors' contributions}

LXL designed data analysis and wrote the manuscript. MLY, XKD, HQL acquired and analysed the data.

YHL edited the manuscript. XMW, AMJ contributed to critical revision. All authors have read and approved the manuscript.

\section{Funding}

No funding was received. Availability of data and materials

\section{Availability of data and materials}

All supporting data can be provided upon request to the authors.

\section{Ethics approval and consent to participate}

This article does not contain any studies with human participants or animals performed by any of the authors.

\section{Consent for publication}

All the authors approved the manuscript.

\section{Competing interests}

The authors declare that they have no competing interests.

\section{Author details}

${ }^{1}$ Department of Spine Surgery, The Third Affiliated Hospital, Sun Yat-sen University, Guangzhou, Guangdong, China. ${ }^{2}$ Department of Joint and Trauma Surgery, The Third Affiliated Hospital of Sun Yatsen University, Guangzhou, Guangdong, China. ${ }^{3}$ Department of Endocrinology, Shenzhen Hospital of Guangzhou University of Chinese Medicine (Futian), Shenzhen, Guangdong, China. ${ }^{4}$ Department of Spine surgery, ZhuJiang Hospital of Southern Medical University, Southern Medical University, Guangzhou, Guangdong, China. ${ }^{5}$ Department of Oncology, Shenzhen Hospital of Guangzhou University of Chinese Medicine (Futian), Shenzhen 518034, Guangdong, China.

\section{References}

1. De la Rosa MB, Kozik EM, Sakaguchi DS. Adult Stem Cell-Based Strategies for Peripheral Nerve Regeneration. Adv Exp Med Biol. 2018;1119:41-71. http://10.1007/5584_2018_254.

2. Neer CS, Grantham SA, Foster RR. Femoral shaft fracture with sciatic nerve palsy. Jama. 1970;214(13):2307-11. 
3. Aufranc OE, Jones WN, Turner RH, Thomas WH. Fracture of acetabulum with dislocation of hip and sciatic palsy. Jama. 1967;201(9):690-1.

4. Jolles BM, Bogoch ER. Posterior versus lateral surgical approach for total hip arthroplasty in adults with osteoarthritis. Cochrane Database Syst Rev. 2006(3):Cd003828. http://10.1002/14651858.CD003828.pub3.

5. Asthana P, Zhang G, Sheikh KA, Him Eddie Ma C. Heat shock protein is a key therapeutic target for nerve repair in autoimmune peripheral neuropathy and severe peripheral nerve injury. Brain, behavior, and immunity. 2021;91:48-64. http://10.1016/j.bbi.2020.08.020.

6. Tallon C, Rockenstein E, Masliah E, Farah MH. Increased BACE1 activity inhibits peripheral nerve regeneration after injury. Neurobiol Dis. 2017;106:147-57. http://10.1016/j.nbd.2017.07.003.

7. Ertürk A, Hellal F, Enes J, Bradke F. Disorganized microtubules underlie the formation of retraction bulbs and the failure of axonal regeneration. J Neurosci. 2007;27(34):9169-80. http://10.1523/jneurosci.0612-07.2007.

8. Qu WR, Zhu Z, Liu J, Song DB, Tian H, Chen BP, et al. Interaction between Schwann cells and other cells during repair of peripheral nerve injury. Neural regeneration research. 2021;16(1):93-8. http://10.4103/1673-5374.286956.

9. Scheib J, Höke A. Advances in peripheral nerve regeneration. Nature reviews Neurology. 2013;9(12):668-76. http://10.1038/nrneurol.2013.227.

10. Christie KJ, Webber CA, Martinez JA, Singh B, Zochodne DW. PTEN inhibition to facilitate intrinsic regenerative outgrowth of adult peripheral axons. J Neurosci. 2010;30(27):9306-15. http://10.1523/jneurosci.6271-09.2010.

11. Coulibaly AP, Isaacson LG. Transient changes in spinal cord glial cells following transection of preganglionic sympathetic axons. Autonomic neuroscience: basic clinical. 2012;168(1-2):32-42. http://10.1016/j.autneu.2012.01.005.

12. Bosse F, Hasenpusch-Theil K, Küry P, Müller HW. Gene expression profiling reveals that peripheral nerve regeneration is a consequence of both novel injury-dependent and reactivated developmental processes. J Neurochem. 2006;96(5):1441-57. http://10.1111/j.1471-4159.2005.03635.x.

13. Palmisano I, Danzi MC, Hutson TH, Zhou L, McLachlan E, Serger E, et al. Epigenomic signatures underpin the axonal regenerative ability of dorsal root ganglia sensory neurons. Nature neuroscience. 2019;22(11):1913-24. http://10.1038/s41593-019-0490-4.

14. Cho Y, Sloutsky R, Naegle KM, Cavalli V. Injury-induced HDAC5 nuclear export is essential for axon regeneration. Cell. 2013;155(4):894-908. http://10.1016/j.cell.2013.10.004.

15. Zhao H, Duan LJ, Sun QL, Gao YS, Yang YD, Tang XS, et al. Identification of Key Pathways and Genes in L4 Dorsal Root Ganglion (DRG) After Sciatic Nerve Injury via Microarray Analysis. Journal of investigative surgery: the official journal of the Academy of Surgical Research. 2020;33(2):17280. http://10.1080/08941939.2018.1452996.

16. Li S, Liu Q, Wang Y, Gu Y, Liu D, Wang C, et al. Differential gene expression profiling and biological process analysis in proximal nerve segments after sciatic nerve transection. PloS one. 
2013;8(2):e57000. http://10.1371/journal.pone.0057000.

17. Langfelder $P$, Horvath $S$. WGCNA: an R package for weighted correlation network analysis. BMC Bioinform. 2008;9:559. http://10.1186/1471-2105-9-559.

18. Zhou J, Guo H, Liu L, Hao S, Guo Z, Zhang F, et al. Construction of co-expression modules related to survival by WGCNA and identification of potential prognostic biomarkers in glioblastoma. J Cell Mol Med. 2021;25(3):1633-44. http://10.1111/jcmm.16264.

19. Esmaeili S, Mehrgou A, Kakavandi N, Rahmati Y. Exploring Kawasaki disease-specific hub genes revealing a striking similarity of expression profile to bacterial infections using weighted gene coexpression network analysis (WGCNA) and co-expression modules identification tool (CEMiTool): An integrated bioinformatics and experimental study. Immunobiology. 2020;225(4):151980. http://10.1016/j.imbio.2020.151980.

20. Feltrin AS, Tahira AC, Simões SN, Brentani H, Martins DC. Assessment of complementarity of WGCNA and NERI results for identification of modules associated to schizophrenia spectrum disorders. PloS one. 2019;14(1):e0210431. http://10.1371/journal.pone.0210431.

21. Mason MJ, Fan G, Plath K, Zhou Q, Horvath S. Signed weighted gene co-expression network analysis of transcriptional regulation in murine embryonic stem cells. BMC Genomics. 2009;10:327. http://10.1186/1471-2164-10-327.

22. Horvath S, Dong J. Geometric interpretation of gene coexpression network analysis. PLoS Comput Biol. 2008;4(8):e1000117. http://10.1371/journal.pcbi.1000117.

23. Horvath S, Zhang B, Carlson M, Lu KV, Zhu S, Felciano RM, et al. Analysis of oncogenic signaling networks in glioblastoma identifies ASPM as a molecular target. Proc Natl Acad Sci U S A. 2006;103(46):17402-7. http://10.1073/pnas.0608396103.

24. Ghazalpour A, Doss S, Zhang B, Wang S, Plaisier C, Castellanos R, et al. Integrating genetic and network analysis to characterize genes related to mouse weight. PLoS Genet. 2006;2(8):e130. http://10.1371/journal.pgen.0020130.

25. Fuller TF, Ghazalpour A, Aten JE, Drake TA, Lusis AJ, Horvath S. Weighted gene coexpression network analysis strategies applied to mouse weight. Mammalian genome: official journal of the International Mammalian Genome Society. 2007;18(6-7):463-72. http://10.1007/s00335-007-90433.

26. Oldham MC, Horvath S, Geschwind DH. Conservation and evolution of gene coexpression networks in human and chimpanzee brains. Proc Natl Acad Sci U S A. 2006;103(47):17973-8. http://10.1073/pnas.0605938103.

27. Szklarczyk D, Franceschini A, Wyder S, Forslund K, Heller D, Huerta-Cepas J, et al. STRING v10: protein-protein interaction networks, integrated over the tree of life. Nucleic acids research. 2015;43(Database issue):D447-52. http://10.1093/nar/gku1003.

28. Shannon P, Markiel A, Ozier O, Baliga NS, Wang JT, Ramage D, et al. Cytoscape: a software environment for integrated models of biomolecular interaction networks. Genome research. 2003;13(11):2498-504. http://10.1101/gr.1239303. 
29. Zhou Y, Zhou B, Pache L, Chang M, Khodabakhshi AH, Tanaseichuk O, et al. Metascape provides a biologist-oriented resource for the analysis of systems-level datasets. Nature communications. 2019;10(1):1523. http://10.1038/s41467-019-09234-6.

30. Jassal B, Matthews L, Viteri G, Gong C, Lorente P, Fabregat A, et al. The reactome pathway knowledgebase. Nucleic acids research. 2020;48(D1):D498-d503. http://10.1093/nar/gkz1031.

31. Fabregat A, Sidiropoulos K, Viteri G, Marin-Garcia P, Ping P, Stein L, et al. Reactome diagram viewer: data structures and strategies to boost performance. Bioinformatics. 2018;34(7):1208-14. http://10.1093/bioinformatics/btx752.

32. Sidiropoulos K, Viteri G, Sevilla C, Jupe S, Webber M, Orlic-Milacic M, et al. Reactome enhanced pathway visualization. Bioinformatics. 2017;33(21):3461-7. http://10.1093/bioinformatics/btx441.

33. Subramanian A, Kuehn H, Gould J, Tamayo P, Mesirov JP. GSEA-P: a desktop application for Gene Set Enrichment Analysis. Bioinformatics. 2007;23(23):3251-3. http://10.1093/bioinformatics/btm369.

34. Cotto KC, Wagner AH, Feng YY, Kiwala S, Coffman AC, Spies G, et al. DGIdb 3.0: a redesign and expansion of the drug-gene interaction database. Nucleic Acids Res. 2018;46(D1):D1068-d1073. http://10.1093/nar/gkx1143.

35. Costigan M, Moss A, Latremoliere A, Johnston C, Verma-Gandhu M, Herbert TA, et al. T-cell infiltration and signaling in the adult dorsal spinal cord is a major contributor to neuropathic pain-like hypersensitivity. The Journal of neuroscience: the official journal of the Society for Neuroscience. 2009;29(46):14415-22. http://10.1523/jneurosci.4569-09.2009.

36. Pennati A, Nylen EA, Duncan ID, Galipeau J. Regulatory B, Cells Normalize. CNS Myeloid Cell Content in a Mouse Model of Multiple Sclerosis and Promote Oligodendrogenesis and Remyelination. J Neurosci. 2020;40(26):5105-15. http://10.1523/jneurosci.2840-19.2020.

37. Curcio M, Bradke F. Axon Regeneration in the Central Nervous System: Facing the Challenges from the Inside. Annu Rev Cell Dev Biol. 2018;34:495-521. http://10.1146/annurev-cellbio-100617062508.

38. Huang TC, Wu HL, Chen SH, Wang YT, Wu CC. Thrombomodulin facilitates peripheral nerve regeneration through regulating M1/M2 switching. J Neuroinflamm. 2020;17(1):240. http://10.1186/s12974-020-01897-z.

39. Neumann E, Küpfer L, Zeilhofer HU. The a2/a3GABAA receptor modulator TPA023B alleviates not only the sensory but also the tonic affective component of chronic pain in mice. Pain. 2020. http://10.1097/j.pain.0000000000002030.

40. Finnerup NB, Kuner R, Jensen TS. Neuropathic pain: From mechanisms to treatment. Physiological reviews. 2020. http://10.1152/physrev.00045.2019.

41. Shutov LP, Warwick CA, Shi X, Gnanasekaran A, Shepherd AJ, Mohapatra DP, et al. The Complement System Component C5a Produces Thermal Hyperalgesia via Macrophage-to-Nociceptor Signaling That Requires NGF and TRPV1. The Journal of neuroscience: the official journal of the Society for Neuroscience. 2016;36(18):5055-70. http://10.1523/jneurosci.3249-15.2016. 
42. Büttner R, Schulz A, Reuter M, Akula AK, Mindos T, Carlstedt A, et al. Inflammaging impairs peripheral nerve maintenance and regeneration. Aging cell. 2018;17(6):e12833. http://10.1111/acel.12833.

43. Deftu AT, Ciorescu R, Gheorghe RO, Mihăilescu D, Ristoiu V. CXCL1 and CXCL2 Inhibit the Axon Outgrowth in a Time- and Cell-Type-Dependent Manner in Adult Rat Dorsal Root Ganglia Neurons. Neurochem Res. 2019;44(9):2215-29. http://10.1007/s11064-019-02861-x.

44. Jiang BC, Liu T, Gao YJ. Chemokines in chronic pain: cellular and molecular mechanisms and therapeutic potential. Pharmacol Ther. 2020;212:107581. http://10.1016/j.pharmthera.2020.107581.

45. Song H, Zhu Z, Zhou Y, Du N, Song T, Liang H, et al. MIF/CD74 axis participates in inflammatory activation of Schwann cells following sciatic nerve injury. J Mol Histol. 2019;50(4):355-67. http://10.1007/s10735-019-09832-0.

46. Sun W, Kou D, Yu Z, Yang S, Jiang C, Xiong D, et al. A Transcriptomic Analysis of Neuropathic Pain in Rat Dorsal Root Ganglia Following Peripheral Nerve Injury. Neuromol Med. 2020;22(2):250-63. http://10.1007/s12017-019-08581-3.

47. Yang JA, He JM, Lu JM, Jie LJ. Jun, Gal. Cd74, and C1 qb as potential indicator for neuropathic pain. Journal of cellular biochemistry. 2018;119(6):4792-8. http://10.1002/jcb.26673.

48. Wang F, Xu S, Shen X, Guo X, Peng Y, Yang J. Spinal macrophage migration inhibitory factor is a major contributor to rodent neuropathic pain-like hypersensitivity. Anesthesiology. 2011;114(3):64359. http://10.1097/ALN.0b013e31820a4bf3.

49. Linnartz-Gerlach B, Bodea LG, Klaus C, Ginolhac A, Halder R, Sinkkonen L, et al. TREM2 triggers microglial density and age-related neuronal loss. Glia. 2019;67(3):539-50. http://10.1002/glia.23563.

50. Mukherjee S, Klaus C, Pricop-Jeckstadt M, Miller JA, Struebing FL. A Microglial Signature Directing Human Aging and Neurodegeneration-Related Gene Networks. Front NeuroSci. 2019;13:2. http://10.3389/fnins.2019.00002.

51. Wang J, Ma SH, Tao R, Xia LJ, Liu L, Jiang YH. Gene expression profile changes in rat dorsal horn after sciatic nerve injury. Neurological research. 2017;39(2):176-82. http://10.1080/01616412.2016.1273590.

52. Klos A, Wende E, Wareham KJ, Monk PN. International Union of Basic and Clinical Pharmacology. [corrected]. LXXXVII. Complement peptide C5a, C4a, and C3a receptors. Pharmacological reviews. 2013;65(1):500-43. http://10.1124/pr.111.005223.

53. Huelsenbeck SC, Rohrbeck A, Handreck A, Hellmich G, Kiaei E, Roettinger I, et al. C3 peptide promotes axonal regeneration and functional motor recovery after peripheral nerve injury. Neurotherapeutics: the journal of the American Society for Experimental NeuroTherapeutics. 2012;9(1):185-98. http://10.1007/s13311-011-0072-y.

54. Polat E, Dağlığlu E, Menekşe G, Dike MS, Özdöl Ç, Türk C, et al. Neuroprotective effects of adalimumab on rats with experimental peripheral nerve injury: An electron microscopic and biochemical study. Ulusal travma ve acil cerrahi dergisi = Turkish. journal of trauma emergency surgery: TJTES. 2016;22(2):134-8. http://10.5505/tjtes.2015.54358. 
55. Smith D, Tweed C, Fernyhough P, Glazner GW. Nuclear factor-kappaB activation in axons and Schwann cells in experimental sciatic nerve injury and its role in modulating axon regeneration: studies with etanercept. J Neuropathol Exp Neurol. 2009;68(6):691-700. http://10.1097/NEN.0b013e3181a7c14e.

56. Stübgen JP. Tumor necrosis factor-alpha antagonists and neuropathy. Muscle Nerve. 2008;37(3):281-92. http://10.1002/mus.20924.

57. Hendry JM, Alvarez-Veronesi MC, Placheta E, Zhang JJ, Gordon T, Borschel GH. ErbB2 blockade with Herceptin (trastuzumab) enhances peripheral nerve regeneration after repair of acute or chronic peripheral nerve injury. Ann Neurol. 2016;80(1):112-26. http://10.1002/ana.24688.

58. Placheta E, Hendry JM, Wood MD, Lafontaine CW, Liu EH, Cecilia Alvarez Veronesi M, et al. The ErbB2 inhibitor Herceptin (Trastuzumab) promotes axonal outgrowth four weeks after acute nerve transection and repair. Neurosci Lett. 2014;582:81-6. http://10.1016/j.neulet.2014.09.006.

\section{Figures}




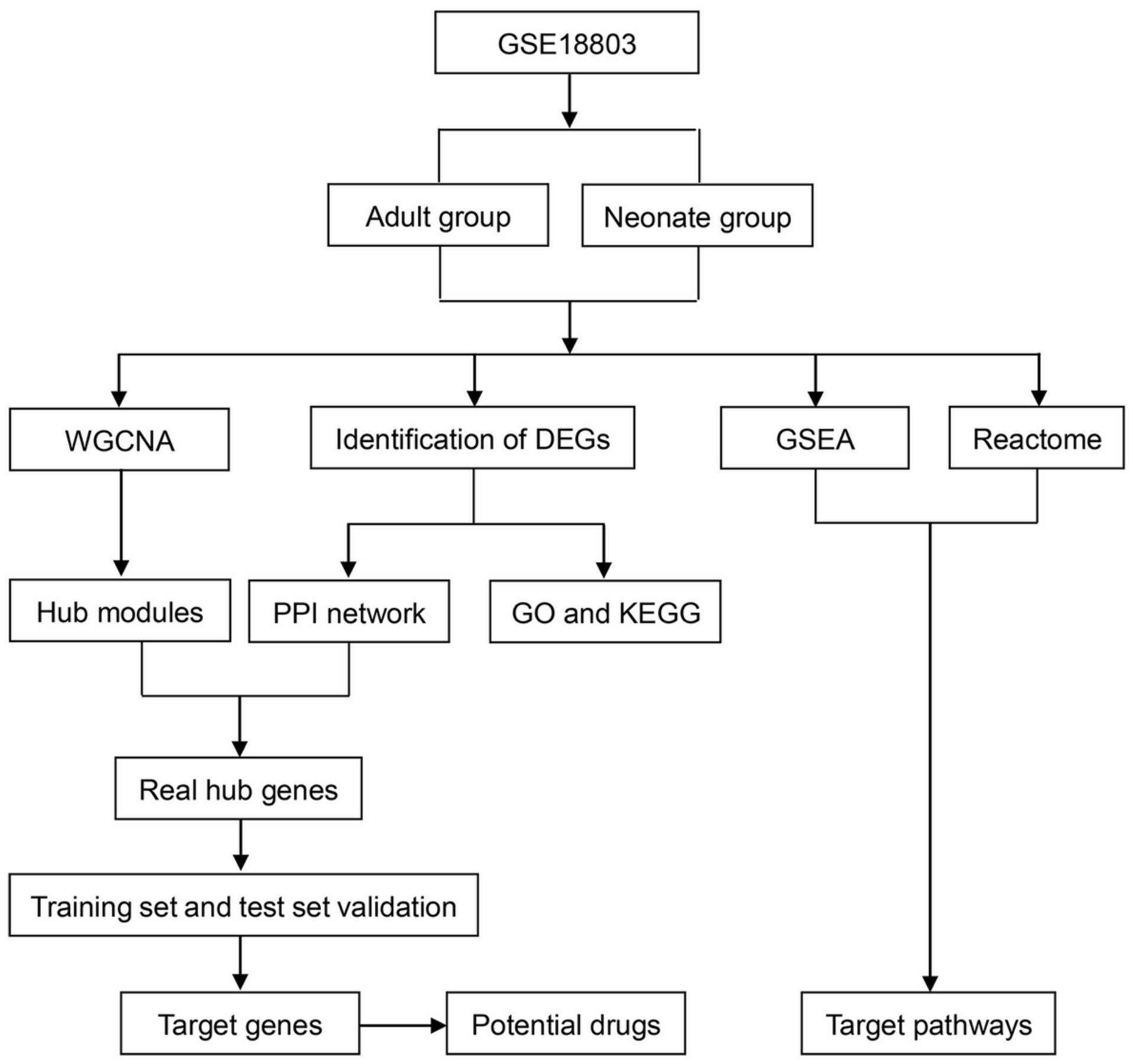

Figure 1

Study flow diagram. 
A

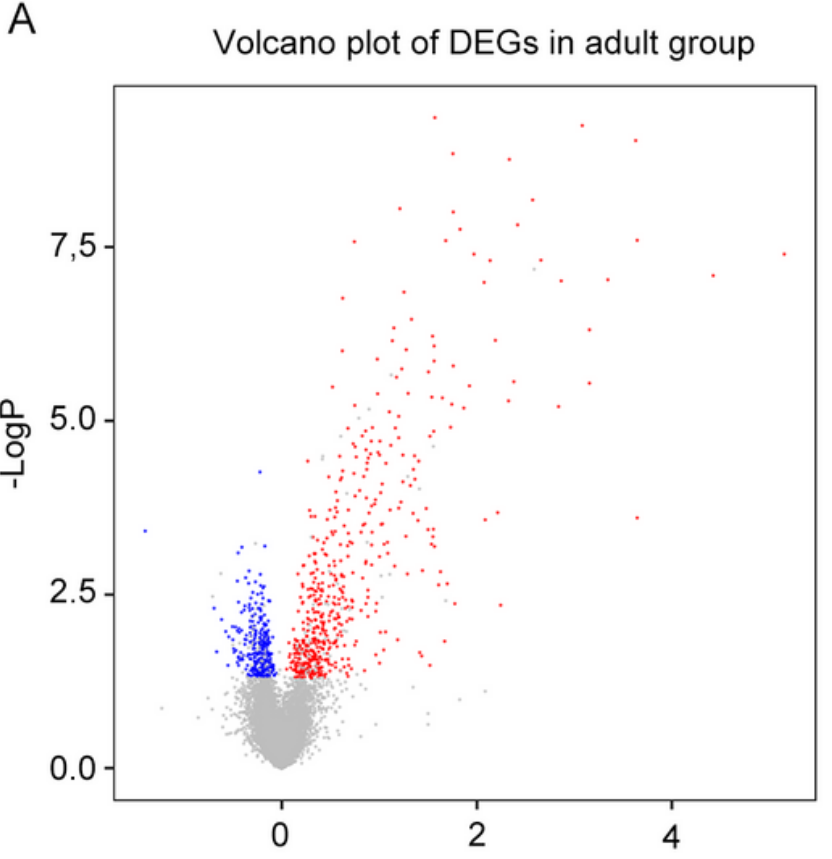

B

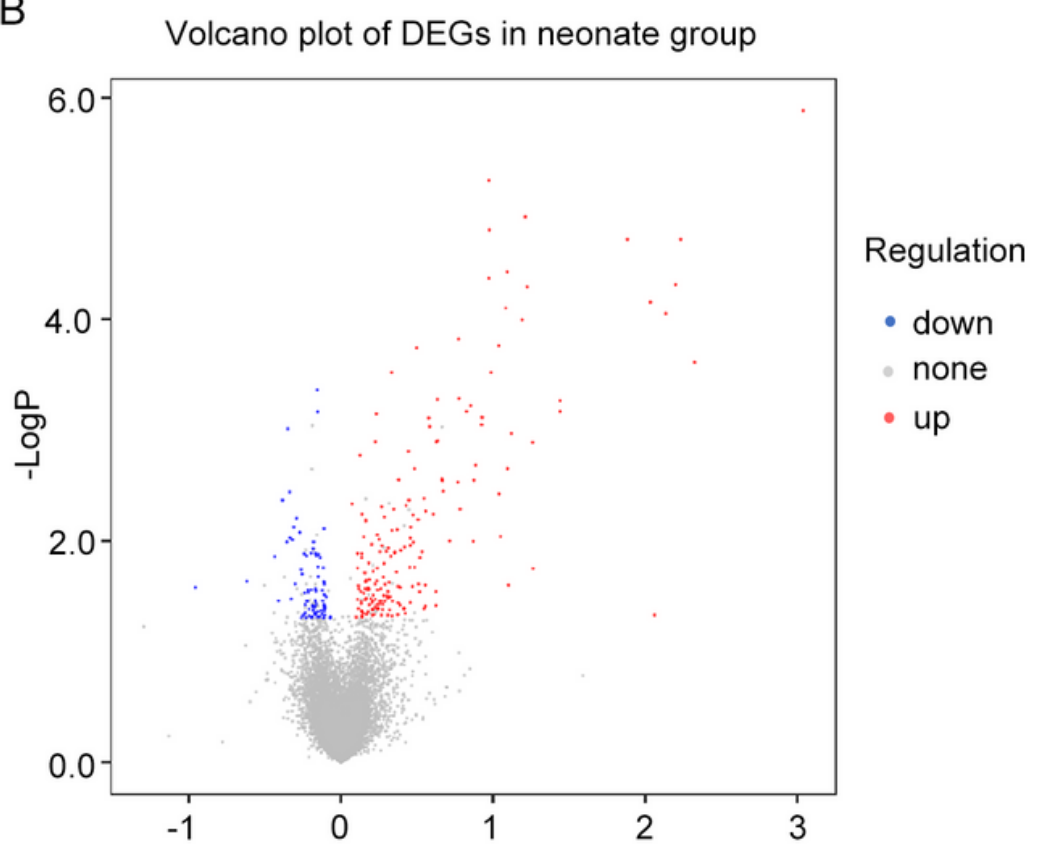

Figure 2

Volcano plot of DEGs. A Adult group. B Neonate group. The red nodes represent upregulated genes selected upon the $|\log 2 \mathrm{FC}| \geq 1.0$ and $\mathrm{P}<0.05$ thresholds, while the blue nodes stand for downregulated genes selected upon the $|\log 2 \mathrm{FC}| \geq 1.0$ and $\mathrm{P}<0.05$ thresholds, and the gray nodes indicate the nonsignificant genes. 
A

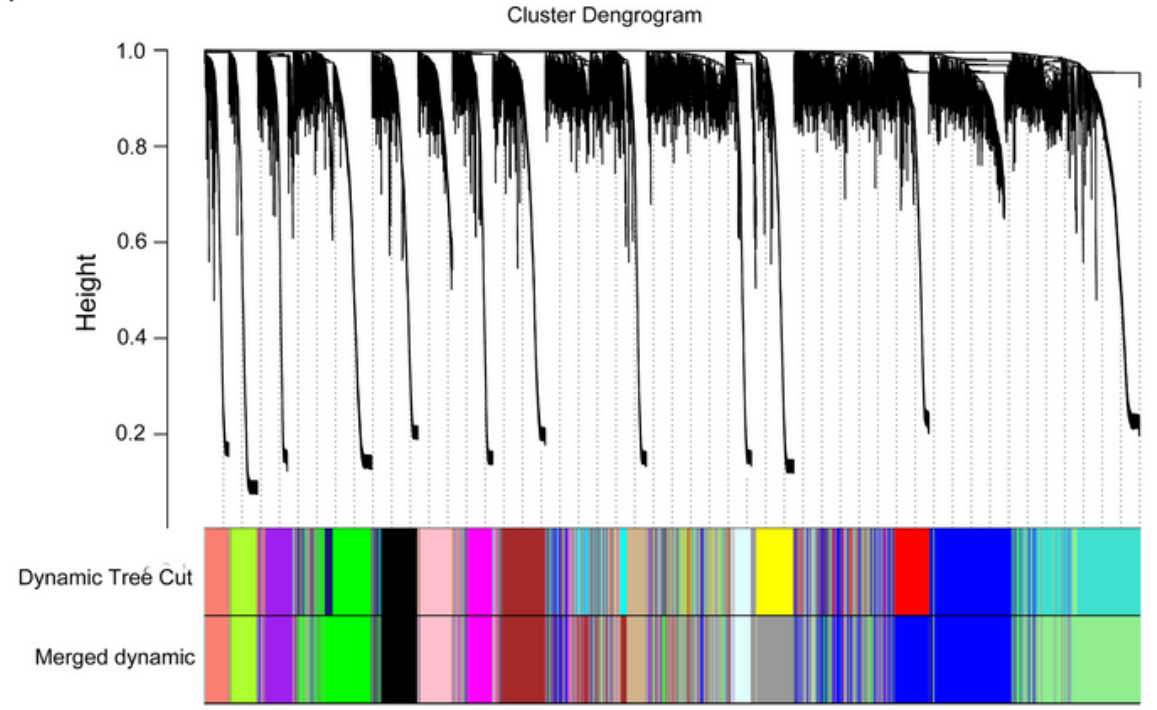

B

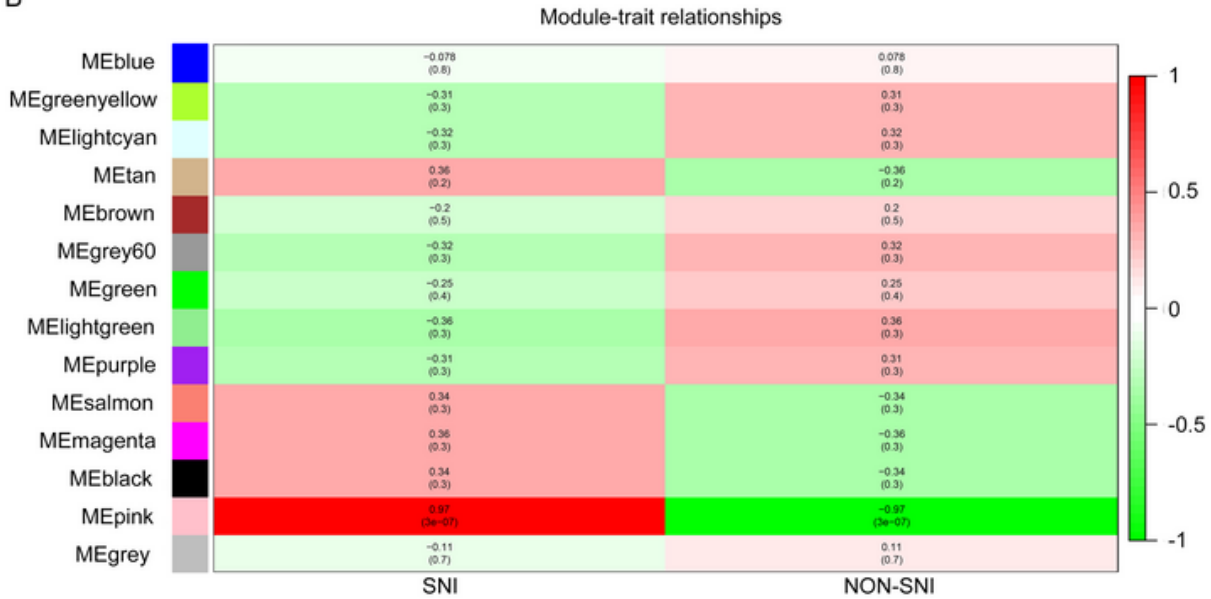

C Eigengene dendrogram

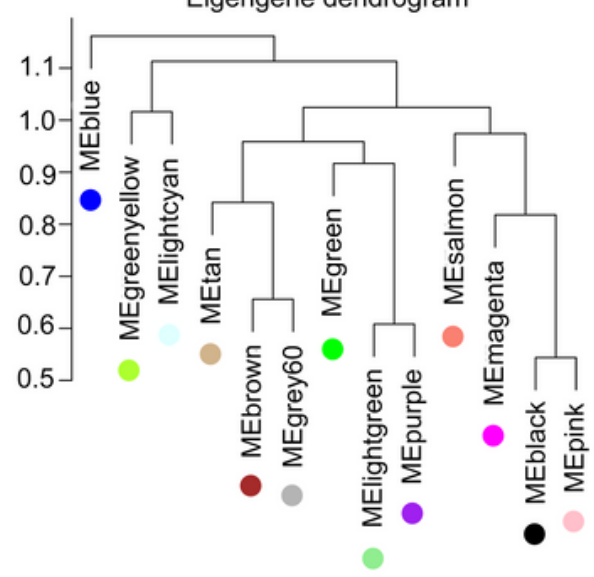

Module membership vs. gene significance cor $=0.88, p=1.6 e-150$

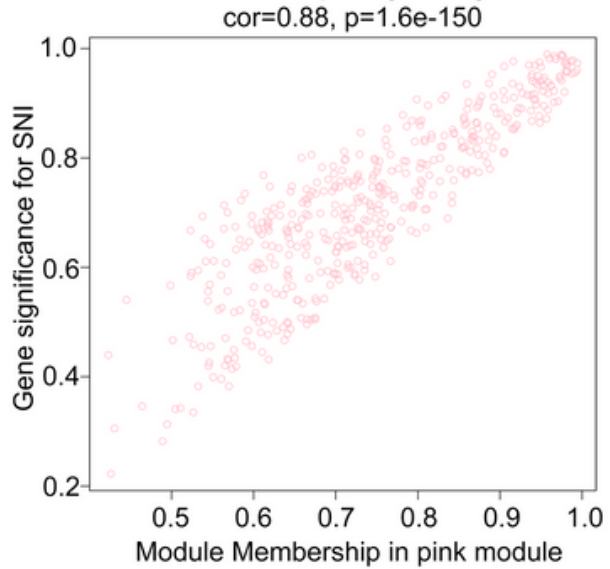

Figure 3

Establishment of weighted co-expression network and analysis of hub modules in adult group. A Dendrogram displaying each DEG clustered in accordance with the dissimilarity measure (1-TOM). As a result, 14 co-expression modules were constructed and were shown in distinctive color. B Heatmap of the relationships of module with the disease traits. In the module, the greater mean gene relevance stands for the greater relationship of this module with the traits of interest. The horizontal and vertical axes stand 
for clinical factors and modules, respectively. The color gradient from red to green represents the shift from positive to negative correlation. The numbers in grids represent correlation coefficients. Values in parenthesis are the P-values for the association test. C System clustering tree for the modules. Dendrogram showing the eigengenes in the consensus module acquired based on WGCNA regarding consensus correlations. D Scatter plot presenting module eigengenes within pink module.

A

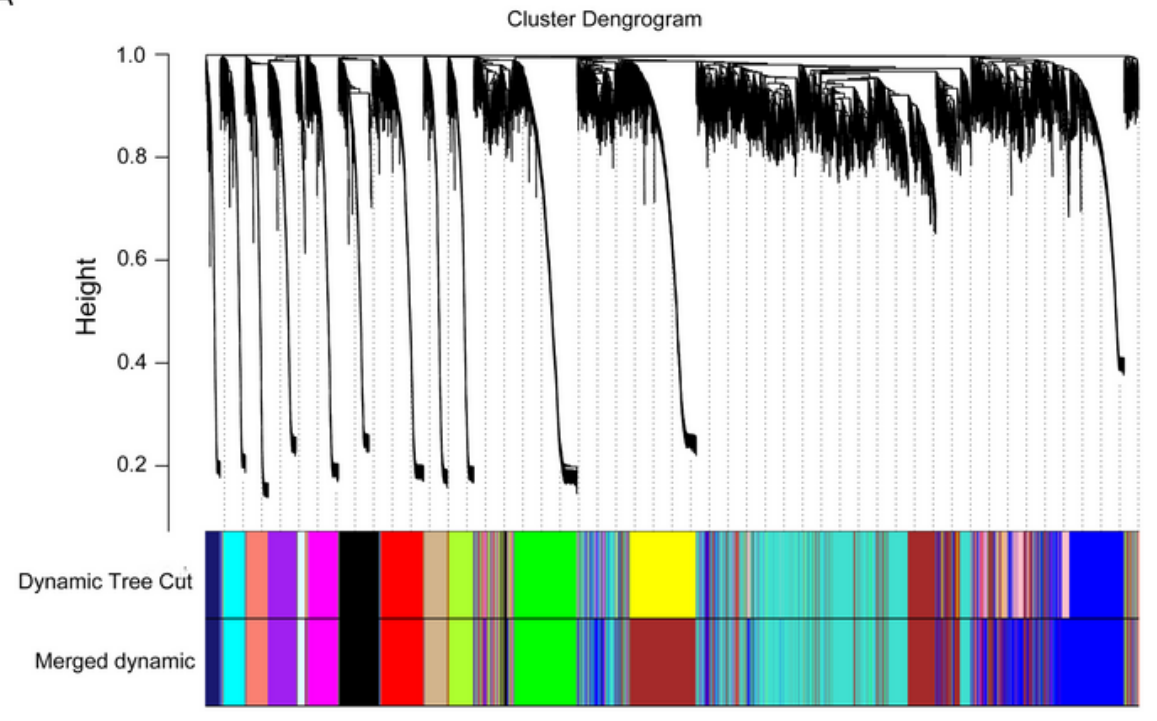

B
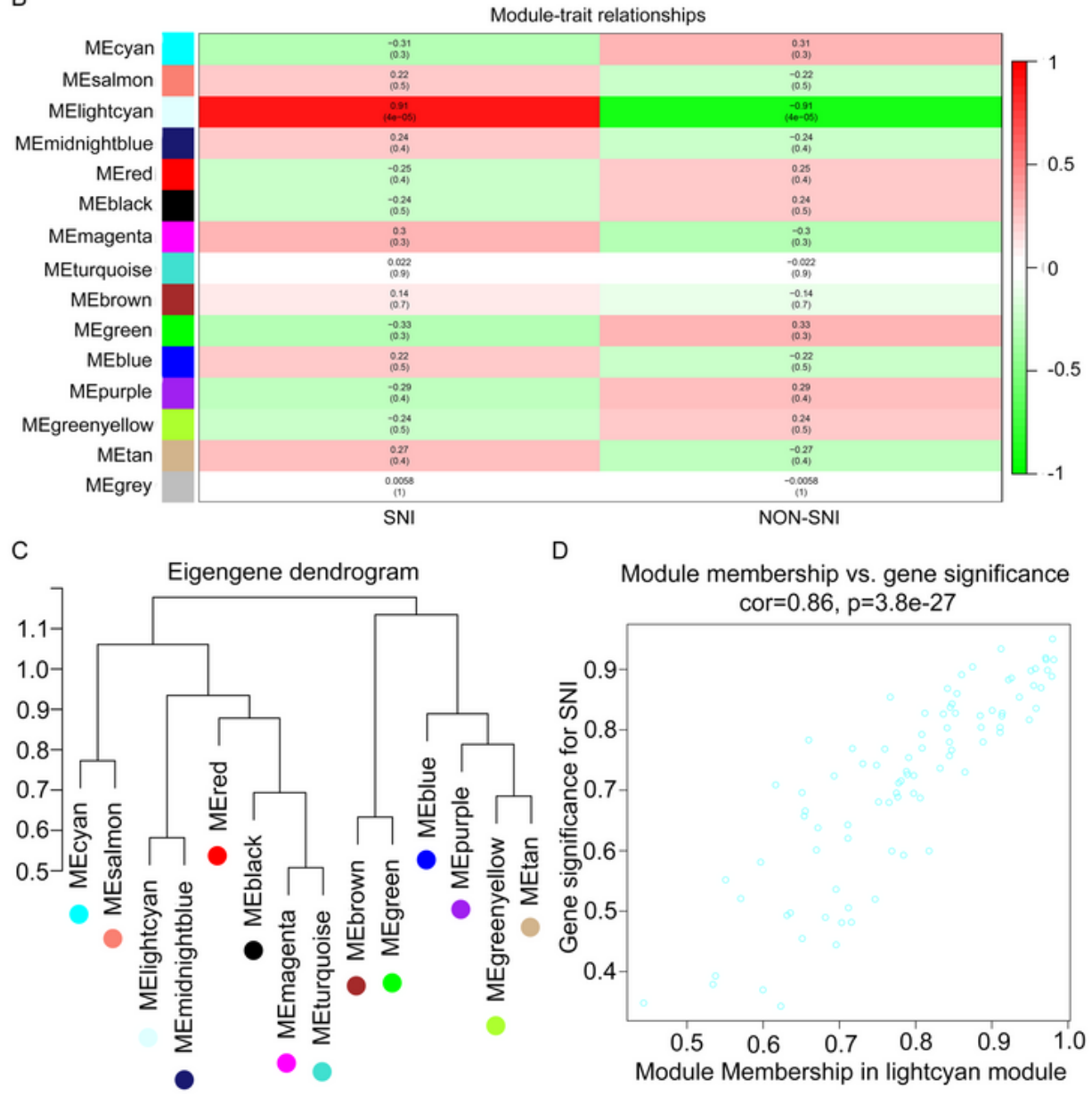

Figure 4 
Establishment of weighted co-expression network and analysis of hub modules in neonate group. A Dendrogram displaying each DEG clustered in accordance with the dissimilarity measure (1-TOM). As a result, 15 co-expression modules were constructed and were shown in distinctive color. B Heatmap of the relationships of module with the disease traits. In the module, the greater mean gene relevance stands for the greater relationship of this module with the traits of interest. The horizontal and vertical axes stand for clinical factors and modules, respectively. The color gradient from red to green represents the shift from positive to negative correlation. The numbers in grids represent correlation coefficients. Values in parenthesis are the P-values for the association test. C System clustering tree for the modules.

Dendrogram showing the eigengenes in the consensus module acquired based on WGCNA regarding consensus correlations. D Scatter plot presenting module eigengenes within lightcyan module.

A

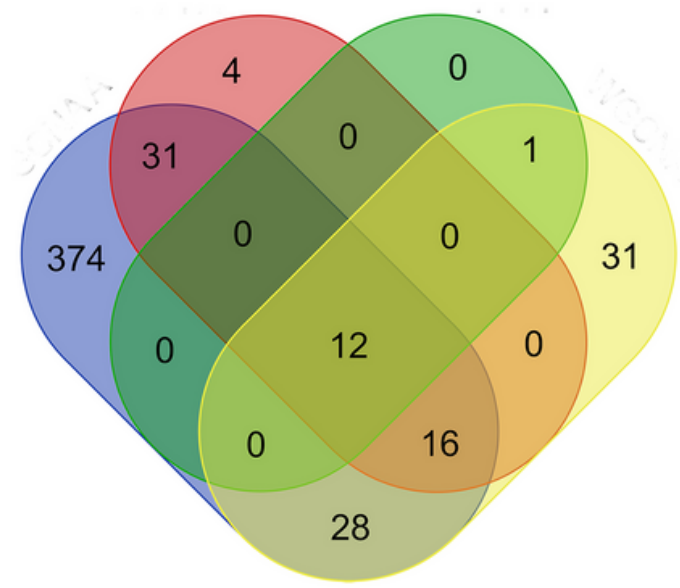

Hub genes in co-expression network in adult group Hub genes in co-expression network in neonate group

Hub genes in PPI network in adult group

Hub genes in PPI network in neonate group
B

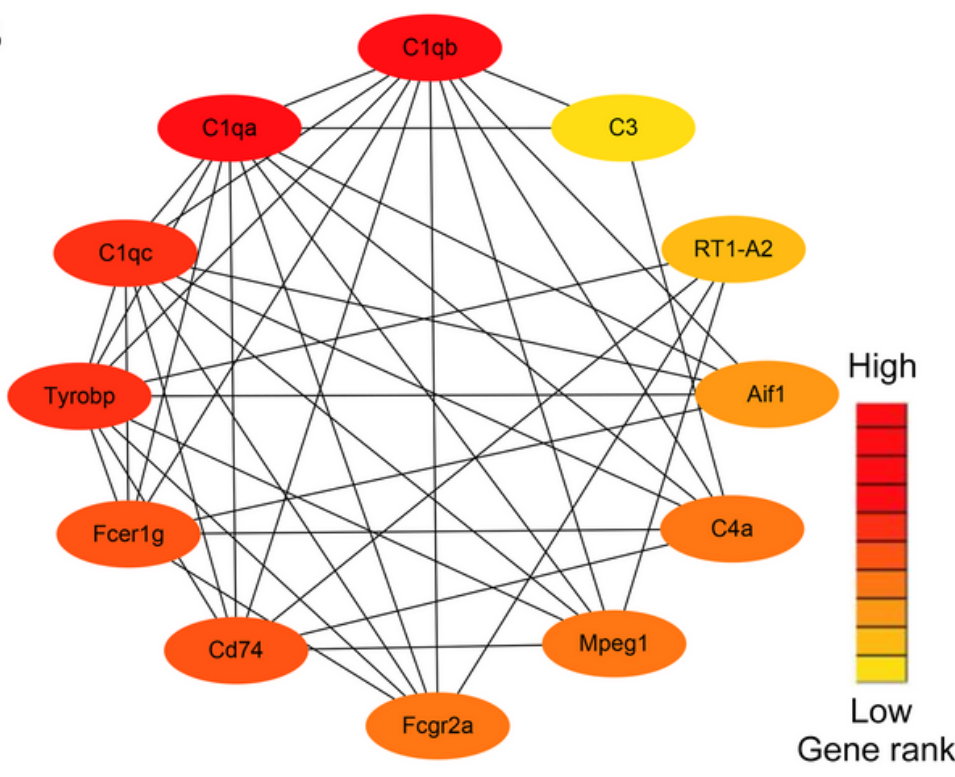

\section{Figure 5}

Detection of hub genes. A A Venn diagram presenting hub genes under co-expression and those involved in the PPI network. B Twelve hub genes (Cd74, C1qb, Tyrobp, C1qa, C4a, RT1-A2, C3, Fcgr2a, Aif1, Fcer1g, Mpeg1, and C1qc) overlapped between the PPI and the co-expression networks. In the heat map, intensity and color of hub genes are shown at right, which represent the gene rank 1 to 12 . 

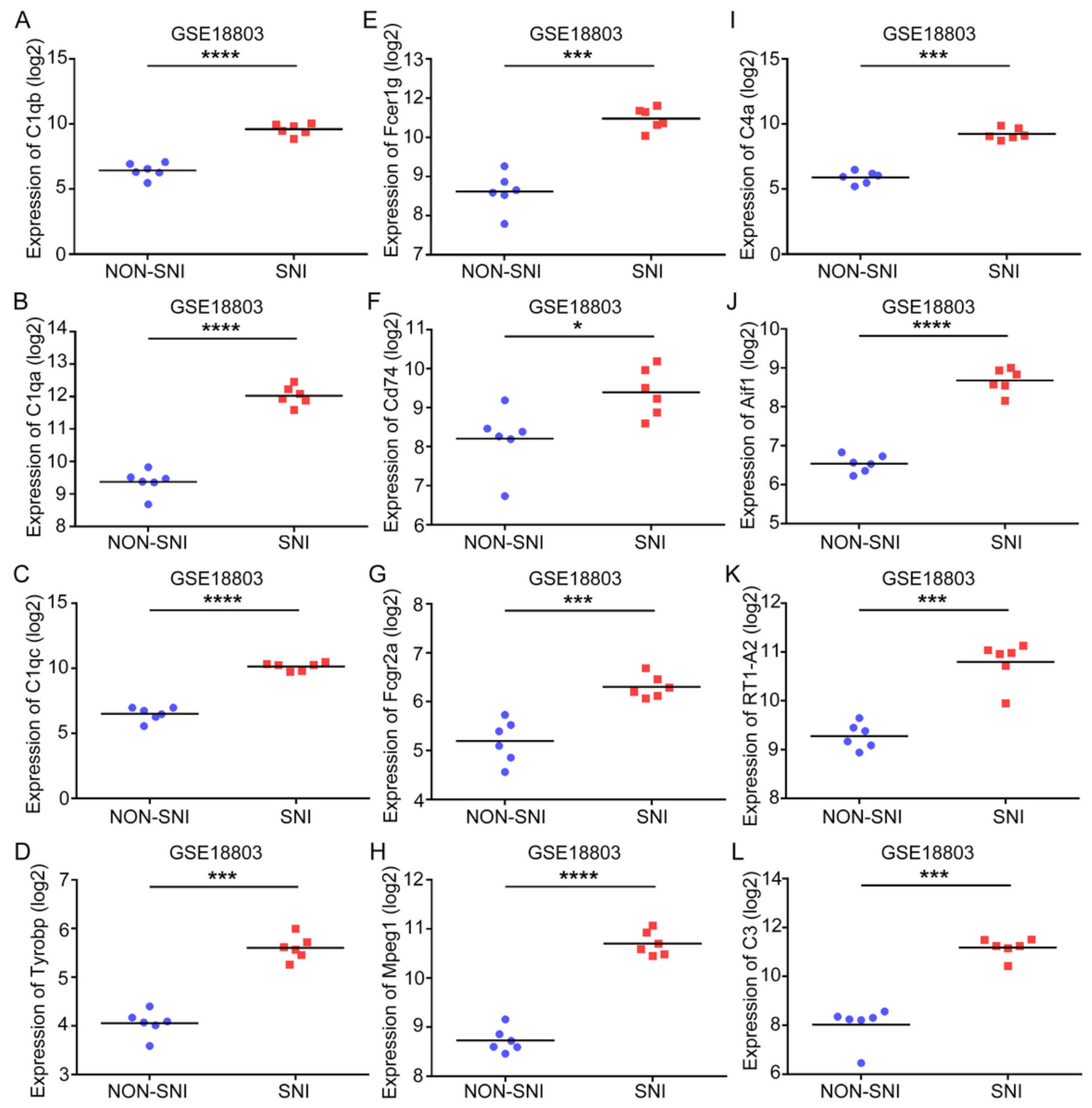

Figure 6

Hub gene validation based on training set (GSE18803). The mRNA level of 12 hub genes was validated in SNI samples compared with normal samples in adult group. All hub genes revealed statistically significant differences in SNI. A C1qb. B C1qa. C C1qc. D Tyrobp. E Fcer1g. F Cd74. G Fcgr2a. H Mpeg1. I C4a. J Aif1. K RT1-A2. L C3. * $P<0.05$, ** $P<0.01$, $* \star * ~ P<0.001$, NS, not significant. 

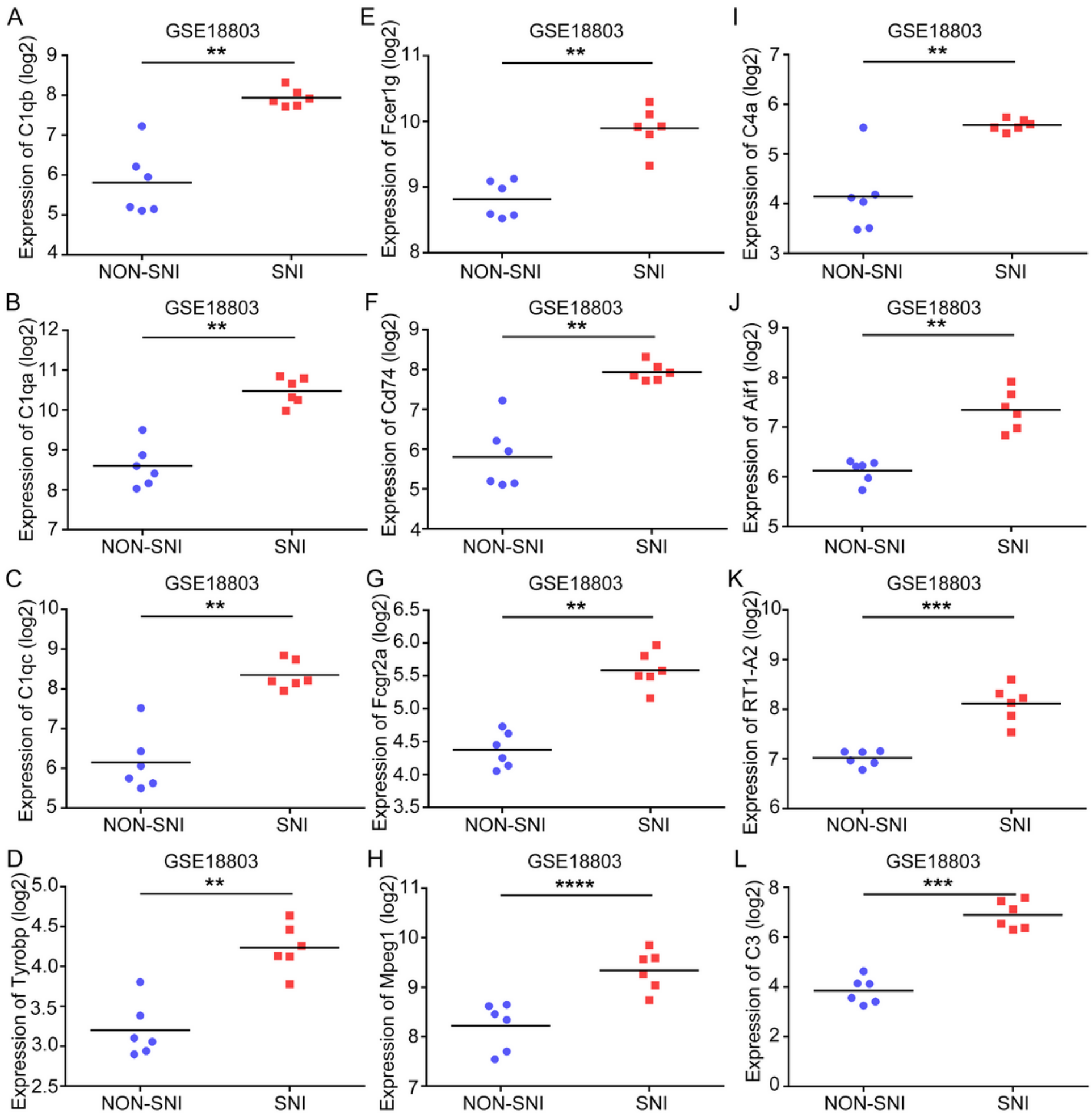

Figure 7

Hub gene validation based on training set (GSE18803). The mRNA level of 12 hub genes was validated in SNI samples compared with normal samples in adult group. All hub genes revealed statistically significant differences in SNI. A C1qb. B C1qa. C C1qc. D Tyrobp. E Fcer1g. F Cd74. G Fcgr2a. H Mpeg1. I C4a. J Aif1. K RT1-A2. L C3. * $P<0.05, * \star P<0.01, * \star * ~ P<0.001$, NS, not significant. 
A

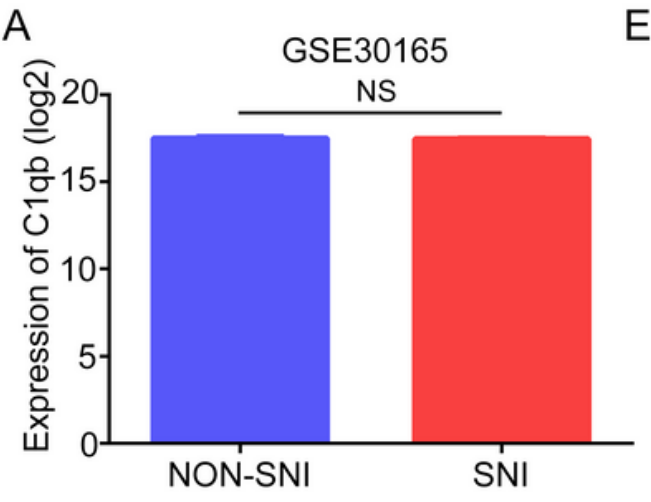

B

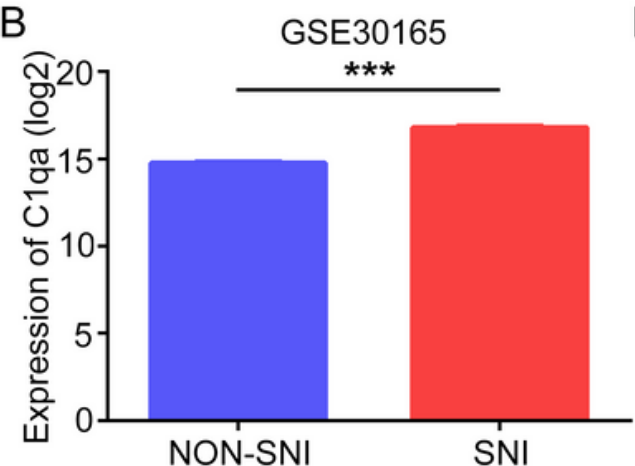

C

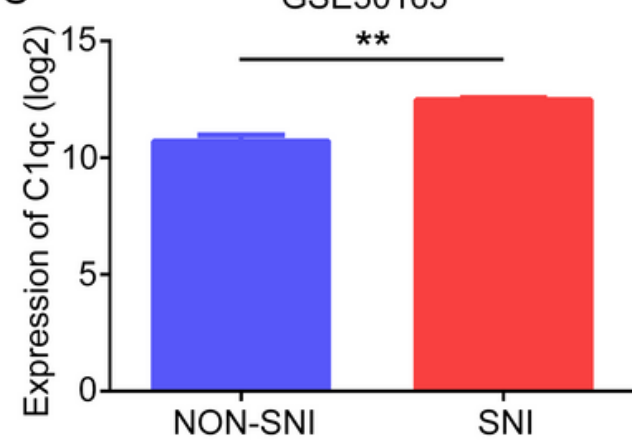

D

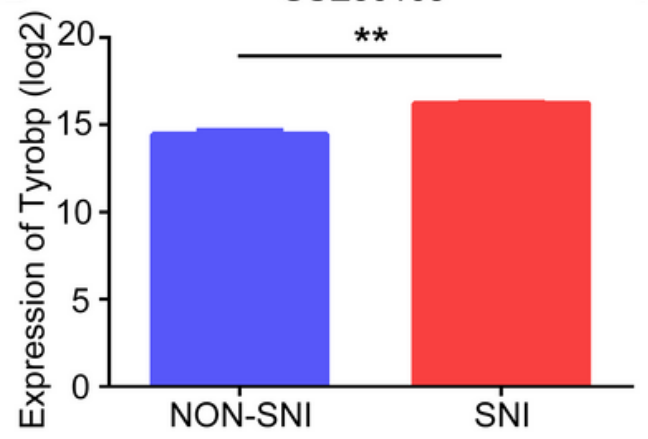

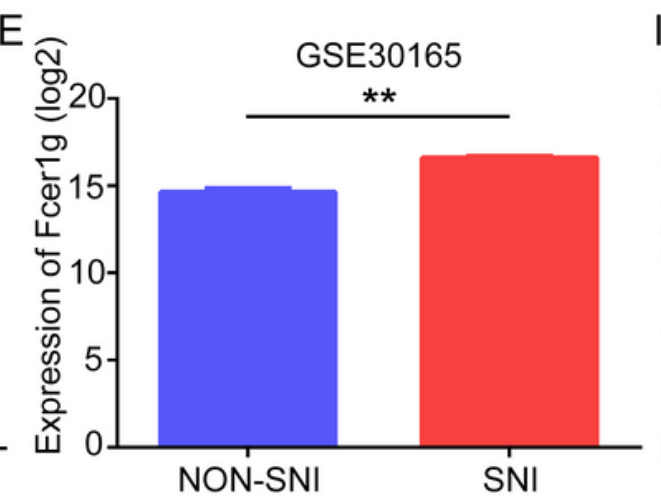

$\mathrm{F}$

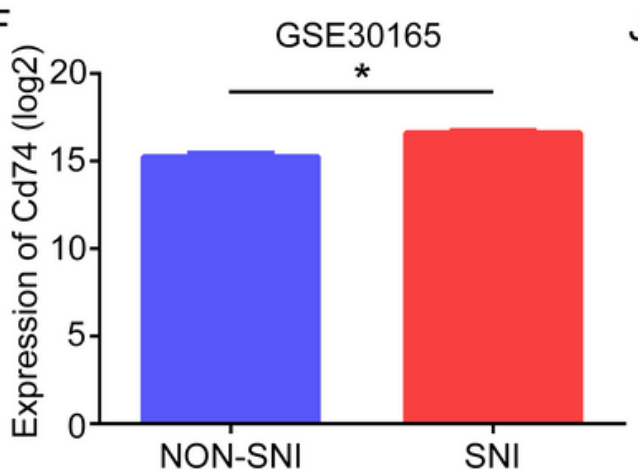

G

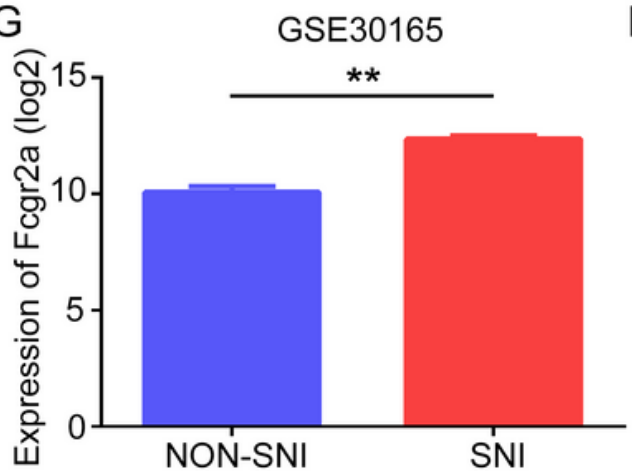

$\mathrm{H}$

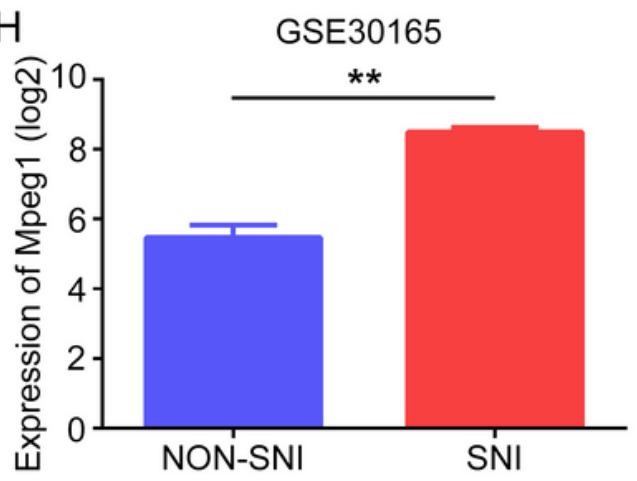

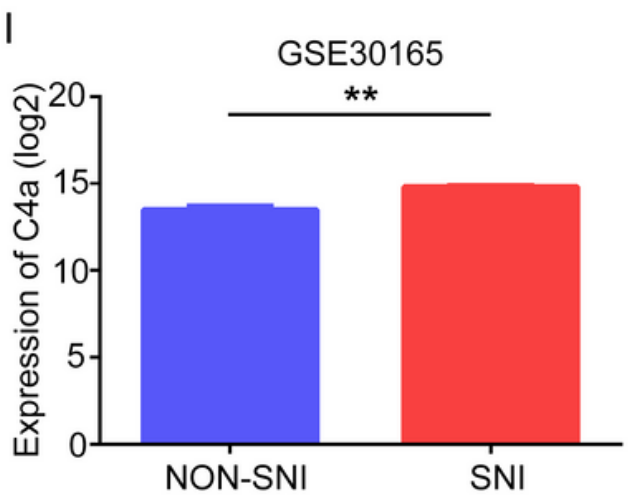

$\mathrm{J}$

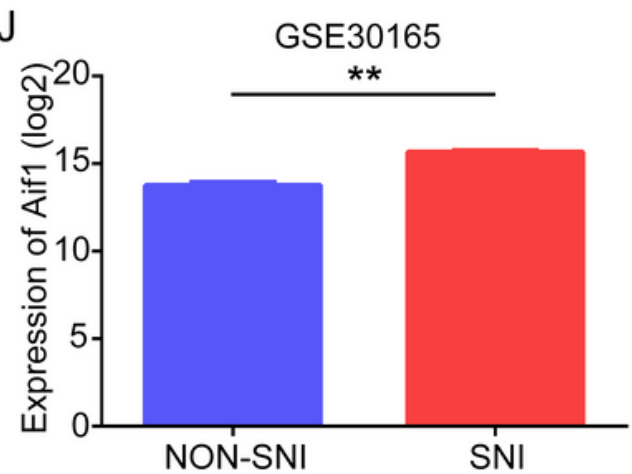

$\mathrm{K}$

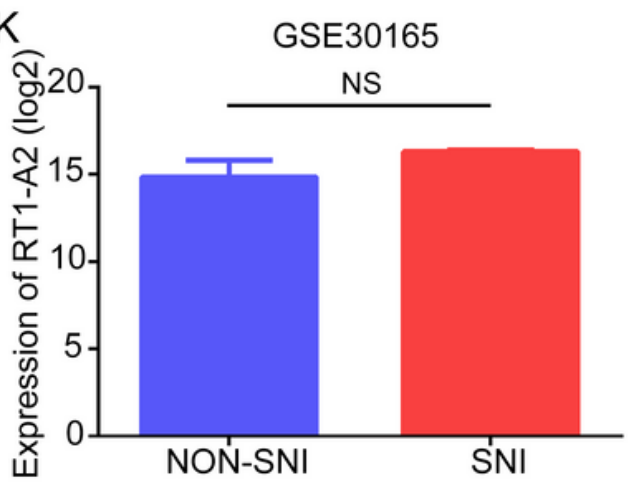

L

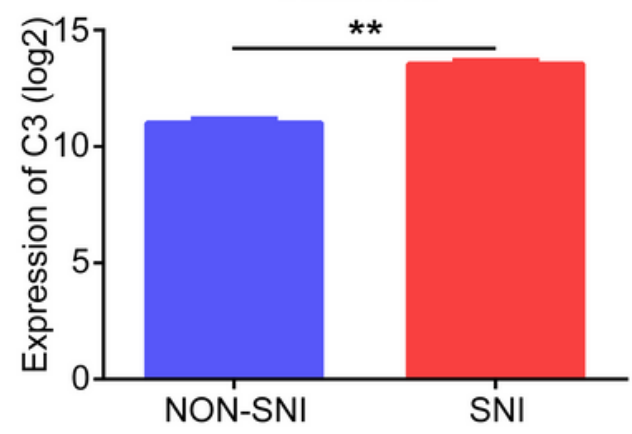

\section{Figure 8}

Hub gene validation based on test set (GSE30165). The mRNA level of 12 hub genes was validated in SNI samples compared with normal samples in neonate group. Ten hub genes were significantly upregulated in SNI samples in comparison to non-SNI samples. A C1qb. B C1qa. C C1qc. D Tyrobp. E Fcer1g. F Cd74. G Fcgr2a. H Mpeg1. I C4a. J Aif1. K RT1-A2. L C3. * $P<0.05$, ** $P<0.01$, $* \star * ~ P<0.001, ~ N S$, not significant. 
A

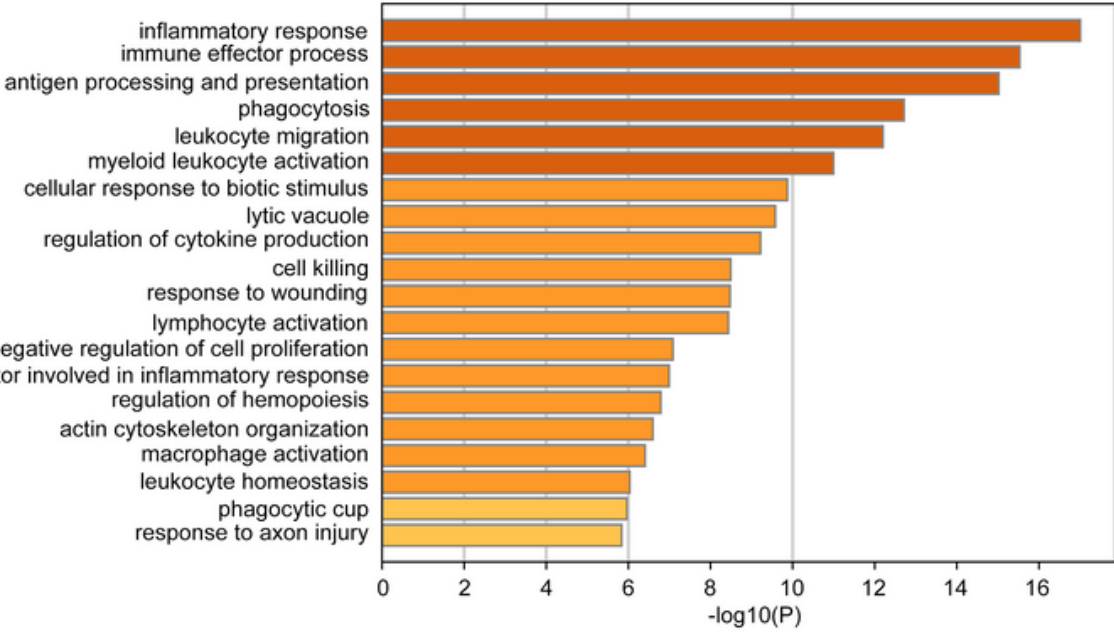

B
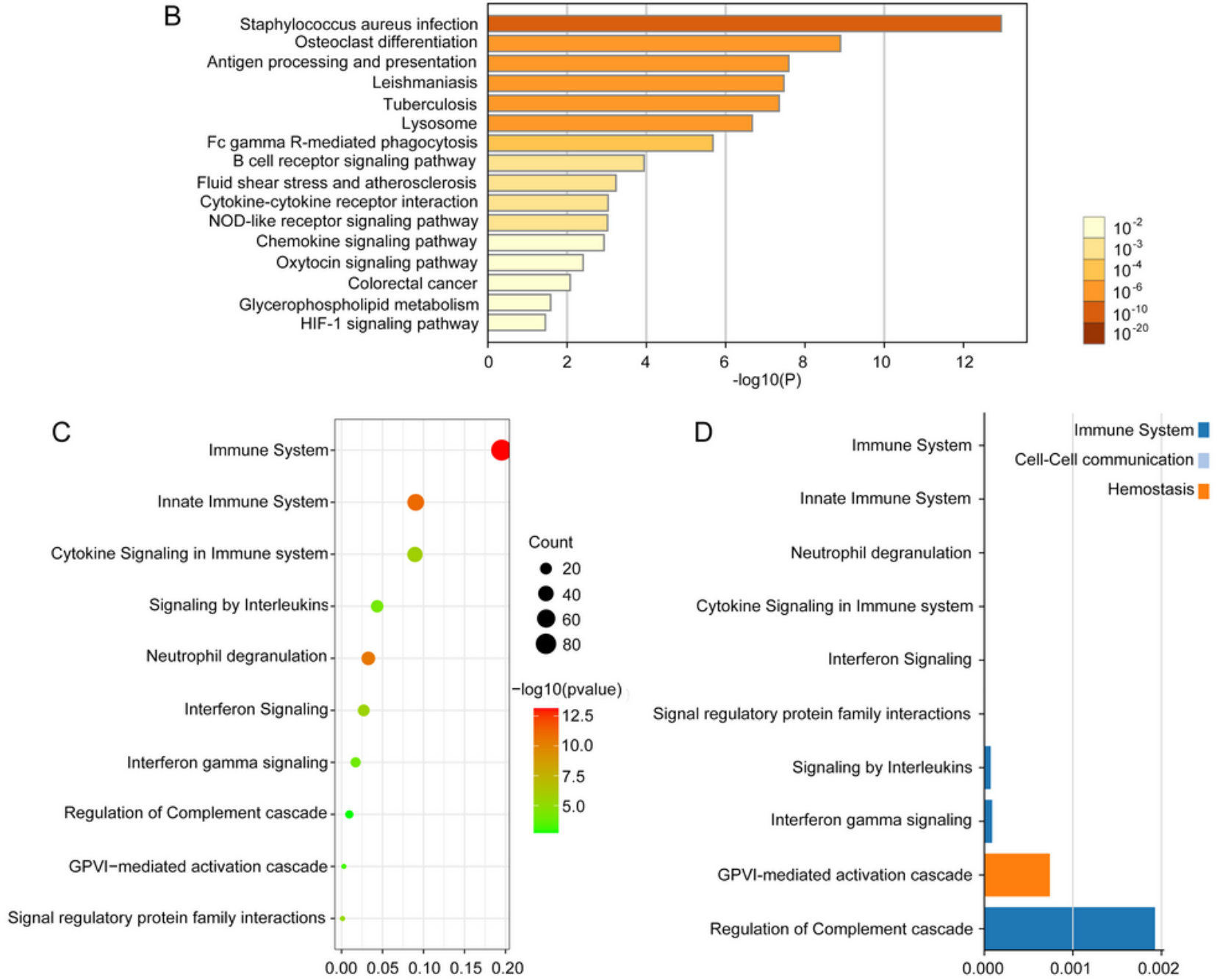

\section{Figure 9}

Potential functional enrichment and pathway analysis of DEGs. A Top 20 clusters functional enrichment of DEGs. B KEGG pathways of DEGs. C The bubble pattern shows the top 10 enrichment pathways with Entities ratio, Entities found (count) and Entities FDR. Y-axis represents pathway name and X-axis represents rich factor. Size and color of each bubble represents the number of differentially expressed genes enriched in the pathway and -log10 (q-value), respectively. D The bar chart demonstrates that the 
gene sets in-volved in metabolism of proteins and signal transduction were significantly enriched in pathways related to $\mathrm{SNI}$ status.
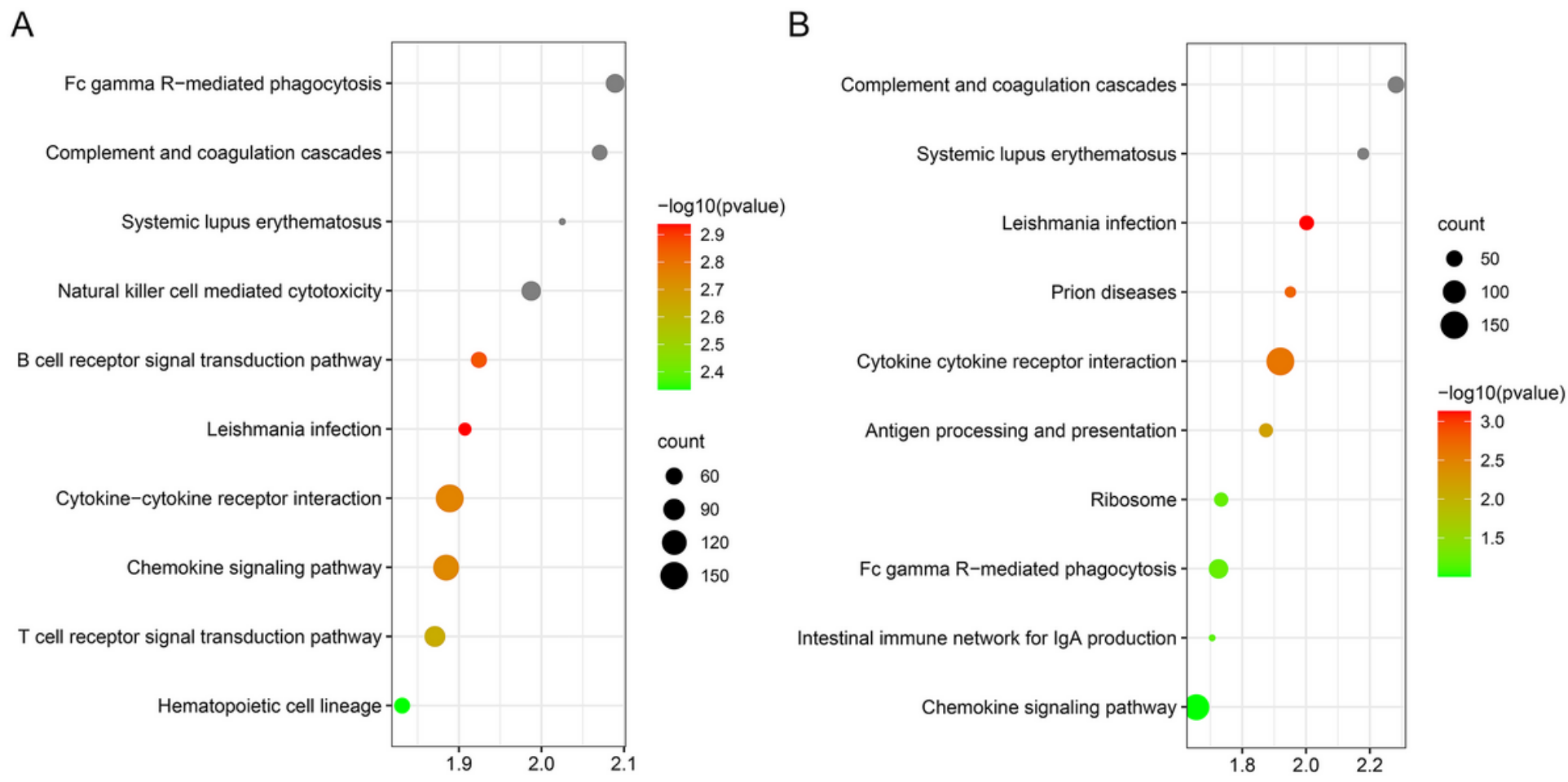

\section{Figure 10}

GSEA. Top 10 functional gene sets enriched in SNI status with high expression of hub genes are shown. A Adult group. B Neonate group. In the expression heatmap figures, the red color stands for upregulated genes, while the blue color indicates the downregulated genes. 
A
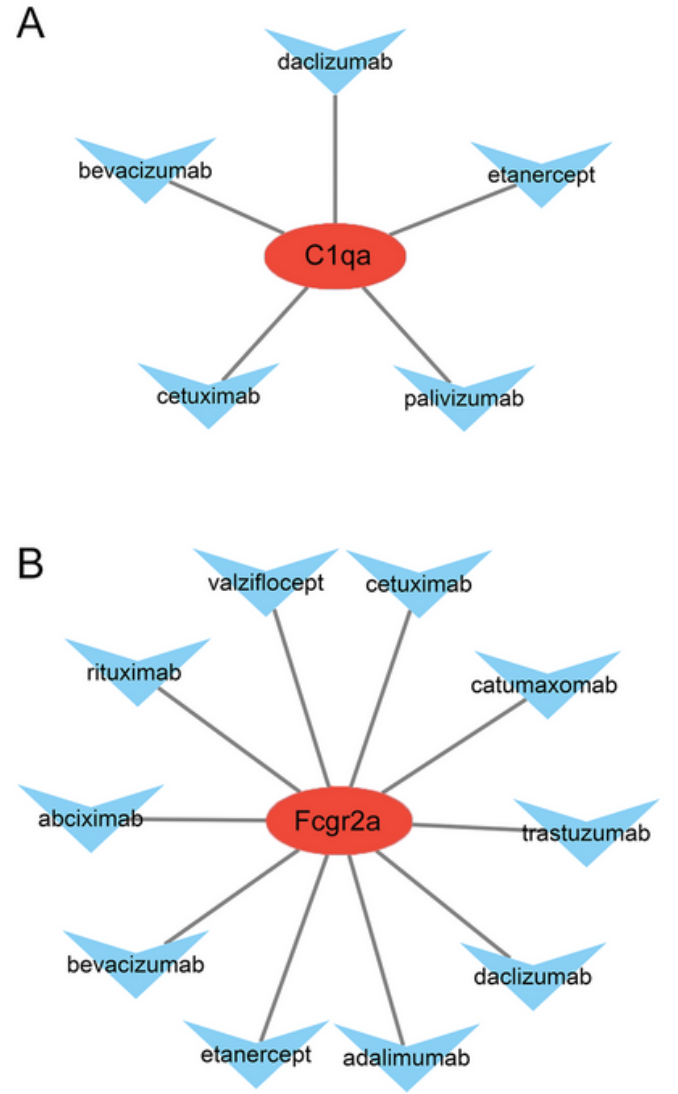

C

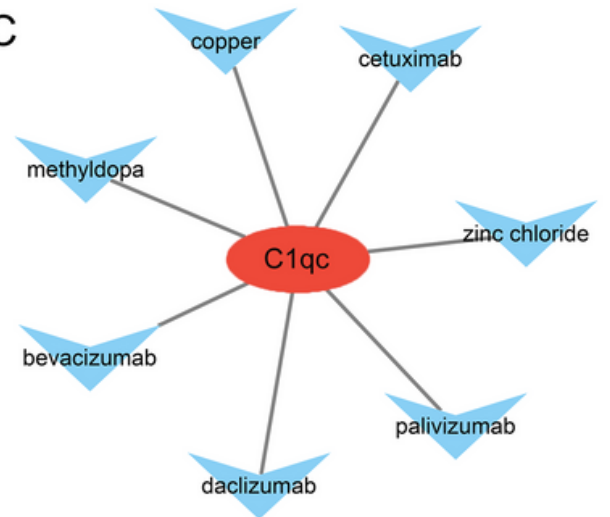

D

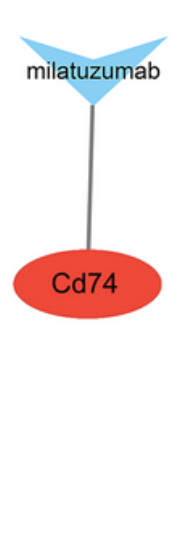

E

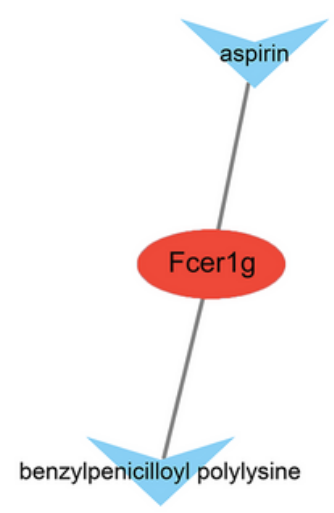

$\mathrm{F}$

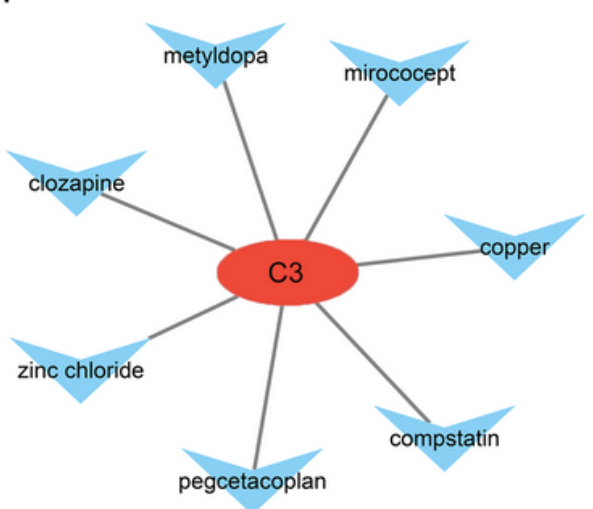

\section{Figure 11}

Drug-gene interaction network. The red ellipse and light-blue $V$ nodes indicate genes and drugs, respectively. A C1qa. B Fcgr2a. C C1qc. D Cd74. E Fcer1g. F C3. 